\title{
AN $h p$ FINITE ELEMENT METHOD TO SOLVE A FLUID-SOLID VIBRATION PROBLEM *
}

\author{
CLAUDIO PADRA ${ }^{\dagger}$, RODOLFO RODRÍGUEZ $\ddagger$, AND MARIO SCHEBLE $\S$
}

\begin{abstract}
This paper deals with a two-dimensional fluid-solid vibration problem arising from nuclear engineering: the vibration of elastically mounted tubes immersed in a cavity filled with fluid. A convenient variational formulation of this problem, valid for compressible and incompressible fluids, is introduced. An $h p$ finite element method is used for its discretization, which leads to a well posed matrix eigenvalue problem. Optimal order a priori error estimates are proved for eigenfunctions and eigenvalues. Then, local a posteriori error indicators are defined and its efficiency and reliability are studied. An adaptive scheme driven by these indicators is proposed and numerically tested.
\end{abstract}

Key words. Fluid structure interaction, vibration problem, finite elements, $h p$ version, spectral approximation, a posteriori error estimates

AMS subject classifications. 65N25, 65N30, 76M10

1. Introduction. The aim of this paper is to introduce and analyze a convenient variational formulation of a fluid-solid vibration problem and an $h p$ finite element adaptive scheme to solve it.

The need of computing the vibration modes of coupled systems involving interaction between fluids and solids arises in many engineering problems. A large amount of work has been devoted to this subject during the last decades; we refer, for instance, to the monographs $[4,10]$, where computer methods and further references are also given. We focus our attention on one of these problems arising from nuclear engineering $([11,12])$ : computing the vibration modes of tubes immersed in a fluid contained in a rigid cavity.

Several models for this problem, which differ from each other on the assumptions on the fluid, have been thoroughly studied in [4]. We consider a homogeneous fluid for which we assume its reference density is constant as well as other usual simplifications for this kind of problems: the viscous effects are not relevant and the velocities are small enough for the convective effects to be neglected. Regarding the tubes, they are assumed to be perfectly rigid, but elastically mounted. Being the tubes parallel, the problem can be posed in a less expensive two-dimensional (2D) framework.

We propose a variational formulation of the problem, which allows for compressible as well as incompressible fluids (it corresponds to what is called in [4] the Laplace and the Helmholtz models, respectively). An important advantage of the proposed formulation is that it leads (after discretization) to a well-posed symmetric matrix generalized eigenvalue problem, which can be safely solved with standard software.

For the discretization we choose an $h p$ finite element method. We show that the resulting discrete problem is well posed and prove spectral approximation properties. In particular, we obtain optimal-order a priori error estimates including a double order of convergence for the eigenvalues.

\footnotetext{
${ }^{*}$ This work was partially supported by ANPCyT (Argentina) under grant PICT-2010-1675.

${ }^{\dagger}$ Centro Atómico Bariloche, 4800, Bariloche, Argentina (padra@cab.cnea.gov.ar). Member of CONICET (Argentina). The work of this author was partially funded by ANPCyT (Argentina) under grant PID-2004-23052.

${ }^{\ddagger} \mathrm{CI}^{2} \mathrm{MA}$, Departamento de Ingeniería Matemática, Universidad de Concepción, Casilla 160-C, Concepción, Chile (rodolfo@ing-mat.udec.cl). The work of this author was partially supported by BASAL project CMM, Universidad de Chile (Chile).

$\S$ Centro Atómico Bariloche, 4800, Bariloche, Argentina (scheble@cab.cnea.gov.ar).
} 
To improve the efficiency of the numerical method, we propose an adaptive scheme based on the $h p$ adaptive strategy from [9]. To drive this scheme, we introduce a posteriori error indicators of residual type, which can be computed locally (and, hence, at low computer cost) from the approximate eigenpair. We analyze the equivalence of the resulting a posteriori estimator with the energy norm of the error. In particular, we prove reliability and efficiency estimates up to higher order terms, the latter with a constant which depends on the polynomial degree of the element. (To the best of the authors' knowledge, simultaneous reliability and efficiency estimates of the energy norm of the error, both with constants independent of the polynomial degree, have not been proved yet for any a posteriori error estimator for $h p$ finite element methods.)

A similar $h p$ adaptive scheme has been recently introduced in [1] for another variational formulation of the same problem valid only for an incompressible fluid. A detailed bibliographic discussion on this topic can be found in this reference; see also the monographs $[5,8]$.

The outline of the paper is as follows. In section 2 , we recall the fluid-structure vibration problem in the case of a compressible fluid and introduce the proposed variational formulation. The $h p$ finite element method is introduced in section 3 and a priori error estimates are proved. These results are extended to an incompressible fluid in section 4. The a posteriori error estimator is introduced in section 5, where reliability and efficiency estimates are proved. Section 6 deals with a couple of implementation issues: the matrix form of the eigenvalue problem and the algorithm of the adaptive scheme. Finally, in section 7, we show how to deal with multiple eigenvalues arising from the symmetry of the problem and report the results of a numerical test which exhibits the performance of the adaptive scheme.

2. The spectral problem. We consider $K$ elastically mounted parallel rigid tubes immersed in a compressible fluid within a rigid cavity. The problem is to determine the free vibration modes of the coupled system.

Under reasonable assumptions (see [4]), this problem can be posed in 2D, a planar transverse section of the cavity being its domain. Only rigid motions of the tubes are allowed. Tube number $i$ is modeled as a harmonic oscillator with stiffness $k_{i}$ and mass $m_{i}$. The fluid is modeled as a potential flow with density $\rho_{0}$ and sound speed $c$.

We denote by $\Omega$ the bounded $2 \mathrm{D}$ domain occupied by the fluid, $\Gamma_{0}$ its outer boundary, and $\Gamma_{i}$ the interface between tube number $i$ and the fluid (see Figure 2.1). We assume that $\Omega$ is a Lipschitz domain with a piecewise smooth boundary (a finite number of angles $\theta \in(0,2 \pi)$ are allowed). Finally, $\boldsymbol{n}$ denotes the unit outer normal to the boundary of $\Omega$.

We write the coupled problem in terms of a fluid velocity potential and the velocities of all rigid tubes. According to [4], the problem of computing the vibration modes of the coupled system reads:

$$
\begin{cases}-\Delta u=\frac{\omega^{2}}{c^{2}} u & \text { in } \Omega, \\ \frac{\partial u}{\partial n}=0 & \text { on } \Gamma_{0}, \\ \frac{\partial u}{\partial n}=\boldsymbol{s}_{i} \cdot \boldsymbol{n} & \text { on } \Gamma_{i}, \quad i=1, \ldots, K, \\ \left(k_{i}-m_{i} \omega^{2}\right) \boldsymbol{s}_{i}=\omega^{2} \rho_{0} \int_{\Gamma_{i}} u \boldsymbol{n} & \text { on } \Gamma_{i}, \quad i=1, \ldots, K,\end{cases}
$$

where $\omega>0$ is the vibration frequency, $u$ is the amplitude of the fluid velocity 


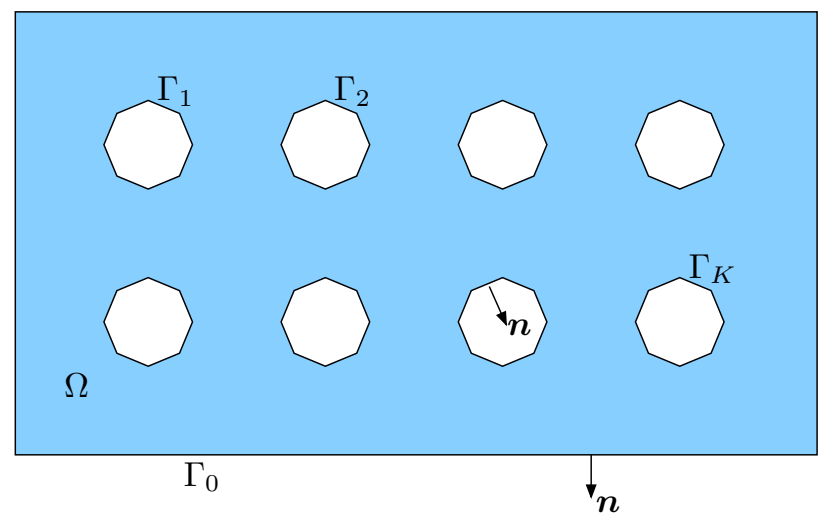

FIG. 2.1. Sketch of the two-dimensional domain

potential and $\boldsymbol{s}_{i}$ the amplitude of the velocity of tube number $i(i=1, \ldots, K)$.

This problem has been studied in [4], where a spectral characterization was obtained and a unitary operator method (cf. [6]) was proposed for its numerical solution. (Notice that $s_{i}$ are used to denote different quantities in [4] and herein; in fact, $\boldsymbol{s}_{i}$ in [4] corresponds to $c^{2}$ times $s_{i}$ herein.) Since our aim is to solve this problem directly by means of a finite element method, our next step is to obtain a convenient alternative variational formulation.

First notice that the velocity potential amplitude $u$ is completely determined by the equations in (2.1) (and not only determined up to an additive constant, as usual). In fact, it follows immediately from the divergence theorem that any solution of this problem with $\omega>0$ satisfies $\int_{\Omega} u=0$. We introduce the spaces $L_{0}^{2}(\Omega):=$ $\left\{\psi \in L^{2}(\Omega): \int_{\Omega} \psi=0\right\}$, endowed with the standard $L^{2}$-norm, and $\mathscr{V}:=H^{1}(\Omega) \cap$ $L_{0}^{2}(\Omega)$. The seminorm $|\cdot|_{H^{1}(\Omega)}$ defines a norm on $\mathscr{V}$ equivalent to the $H^{1}(\Omega)$-norm.

To obtain a variational form of problem (2.1), we multiply the first equation by a test function in $\mathscr{V}$, integrate by parts, and use the third equation to write

$$
\int_{\Omega} \nabla u \cdot \nabla v=\frac{\omega^{2}}{c^{2}} \int_{\Omega} u v+\sum_{i=1}^{K} \boldsymbol{s}_{i} \cdot\left(\int_{\Gamma_{i}} v \boldsymbol{n}\right) \quad \forall v \in \mathscr{V} .
$$

Then, we rewrite the fourth equation as follows:

$$
\boldsymbol{s}_{i}=\omega^{2}\left(\frac{\rho_{0}}{k_{i}} \int_{\Gamma_{i}} u \boldsymbol{n}+\frac{m_{i}}{k_{i}} \boldsymbol{s}_{i}\right), \quad i=1, \ldots, K .
$$

By substituting (2.3) into (2.2), we are lead to

$$
\int_{\Omega} \nabla u \cdot \nabla v=\omega^{2}\left\{\frac{1}{c^{2}} \int_{\Omega} u v+\sum_{i=1}^{K} \frac{\rho_{0}}{k_{i}}\left[\left(\int_{\Gamma_{i}} u \boldsymbol{n}\right) \cdot\left(\int_{\Gamma_{i}} v \boldsymbol{n}\right)+\frac{m_{i}}{k_{i}} \boldsymbol{s}_{i} \cdot\left(\int_{\Gamma_{i}} v \boldsymbol{n}\right)\right]\right\} .
$$

The final step is to write (2.3) in a convenient weak form to obtain a final symmetric problem:

$$
\sum_{i=1}^{K} \frac{m_{i}}{\rho_{0}} \boldsymbol{s}_{i} \cdot \boldsymbol{t}_{i}=\omega^{2} \sum_{i=1}^{K}\left[\frac{m_{i}}{k_{i}}\left(\int_{\Gamma_{i}} u \boldsymbol{n}\right) \cdot \boldsymbol{t}_{i}+\frac{m_{i}^{2}}{\rho_{0} k_{i}} \boldsymbol{s}_{i} \cdot \boldsymbol{t}_{i}\right] \quad \forall \boldsymbol{t}:=\left(\begin{array}{c}
\boldsymbol{t}_{1} \\
\vdots \\
\boldsymbol{t}_{K}
\end{array}\right) \in \mathbb{R}^{2 K} .
$$


Thus, after some elementary algebraic manipulation, we obtain the following variational form of problem (2.1):

Find $\omega>0$ and non-vanishing $(u, s) \in \mathscr{V} \times \mathbb{R}^{2 K}$ such that

$$
a((u, \boldsymbol{s}),(v, \boldsymbol{t}))=\omega^{2} b((u, \boldsymbol{s}),(v, \boldsymbol{t})) \quad \forall(v, \boldsymbol{t}) \in \mathscr{V} \times \mathbb{R}^{2 K},
$$

where $a$ and $b$ are the bilinear forms defined as follows:

$$
\begin{aligned}
& a((u, \boldsymbol{s}),(v, \boldsymbol{t})):=\int_{\Omega} \nabla u \cdot \nabla v+\sum_{i=1}^{K} \frac{m_{i}}{\rho_{0}} \boldsymbol{s}_{i} \cdot \boldsymbol{t}_{i} \\
& b((u, \boldsymbol{s}),(v, \boldsymbol{t})):=\frac{1}{c^{2}} \int_{\Omega} u v+\sum_{i=1}^{K} \frac{\rho_{0}}{k_{i}}\left(\int_{\Gamma_{i}} u \boldsymbol{n}+\frac{m_{i}}{\rho_{0}} \boldsymbol{s}_{i}\right) \cdot\left(\int_{\Gamma_{i}} v \boldsymbol{n}+\frac{m_{i}}{\rho_{0}} \boldsymbol{t}_{i}\right) .
\end{aligned}
$$

Both bilinear forms are symmetric, continuous, and elliptic on $\mathscr{X}:=\mathscr{V} \times \mathbb{R}^{2 K}$. (Let us remark that, because of the symmetry, it makes sense to pose the spectral problem in terms of real eigenvalues and eigenfunctions.) We endow $\mathscr{X}$ with the norm $\|\cdot\|_{a}$ induced by the inner product $a(\cdot, \cdot)$. We also denote by $\|\cdot\|_{b}$ the norm on $\mathscr{X}$ induced by $b(\cdot, \cdot)$.

Remark 2.1. Each solution of (2.4) satisfies also this equation for all test functions $(v, \boldsymbol{t}) \in H^{1}(\Omega) \times \mathbb{R}^{2 K}$. This follows from the facts that $H^{1}(\Omega)=\mathscr{V} \oplus \mathbb{R}$ and that, for $v$ constant, $a((u, s),(v, \mathbf{0}))=0$ and $b((u, s),(v, \mathbf{0}))=0$, the latter because $\int_{\Omega} u=0$ for $u \in \mathscr{V}$.

Notice that problem (2.4) is clearly equivalent to (2.2)-(2.3). The previous remark allows us to show that the variational spectral problem (2.4) is also equivalent to (2.1).

The aim of this paper is to introduce and analyze an $h p$ finite element method to numerically solve problem (2.4). For the analysis, we will apply the classical spectral approximation theory from [2]. With this purpose, we introduce the corresponding solution operator $T: \mathscr{X} \rightarrow \mathscr{X}$ defined for each $(f, \boldsymbol{q}) \in \mathscr{X}$ by $T(f, \boldsymbol{q}):=(w, \boldsymbol{r})$, where $(w, \boldsymbol{r}) \in \mathscr{X}$ is the unique solution of the associated source problem

$$
a((w, \boldsymbol{r}),(v, \boldsymbol{t}))=b((f, \boldsymbol{q}),(v, \boldsymbol{t})) \quad \forall(v, \boldsymbol{t}) \in \mathscr{V} \times \mathbb{R}^{2 K} .
$$

It is immediate to show that the unique solution of this problem also satisfies

$$
\int_{\Omega} \nabla w \cdot \nabla v=\frac{1}{c^{2}} \int_{\Omega} f v+\sum_{i=1}^{K}\left(\frac{\rho_{0}}{k_{i}} \int_{\Gamma_{i}} f \boldsymbol{n}+\frac{m_{i}}{k_{i}} \boldsymbol{q}_{i}\right) \cdot\left(\int_{\Gamma_{i}} v \boldsymbol{n}\right) \quad \forall v \in \mathscr{V}
$$

and

$$
\boldsymbol{r}_{i}=\frac{\rho_{0}}{k_{i}} \int_{\Gamma_{i}} f \boldsymbol{n}+\frac{m_{i}}{k_{i}} \boldsymbol{q}_{i}, \quad i=1, \ldots, K
$$

Moreover, by substituting (2.7) into the previous equation, we obtain

$$
\int_{\Omega} \nabla w \cdot \nabla v=\frac{1}{c^{2}} \int_{\Omega} f v+\sum_{i=1}^{K} \boldsymbol{r}_{i} \cdot\left(\int_{\Gamma_{i}} v \boldsymbol{n}\right) \quad \forall v \in \mathscr{V}
$$

Hence, because of a standard a priori estimate for the Neumann problem (see [7]), we know that there exists $r>0$ such that $w \in H^{1+r}(\Omega)$ and

$$
\|w\|_{H^{1+r}(\Omega)} \leq C\left[\frac{1}{c^{2}}\|f\|_{L^{2}(\Omega)}^{2}+\sum_{i=1}^{K}\left\|\boldsymbol{r}_{i} \cdot \boldsymbol{n}\right\|_{L^{2}\left(\Gamma_{i}\right)}^{2}\right]^{1 / 2} .
$$


On the other hand, from (2.7),

$$
\left\|\boldsymbol{r}_{i} \cdot \boldsymbol{n}\right\|_{L^{2}\left(\Gamma_{i}\right)}^{2} \leq C\left(\|f\|_{H^{1}(\Omega)}^{2}+\left|\boldsymbol{q}_{i}\right|^{2}\right), \quad i=1, \ldots K,
$$

so that, because of Poincaré inequality,

$$
\|w\|_{H^{1+r}(\Omega)} \leq C\|(f, \boldsymbol{q})\|_{a} .
$$

Consequently, $T$ is a compact operator. Moreover, because of the symmetry of $a$ and $b, T$ is self-adjoint with respect to any of these inner products. Thus its spectrum reduces to a sequence of finite-multiplicity eigenvalues converging to 0 , the eigenvalues being positive because of the ellipticity of both bilinear forms. It is clear that the non-zero eigenvalues of $T$ are the reciprocals of the eigenvalues of problem (2.4) and the corresponding eigenfrequencies coincide. This yields a thorough spectral characterization of problem (2.1) because of the equivalence between both problems. Let us remark that such characterization was also obtained in [4] by means of a slightly different associated operator.

In the following section, we will analyze a finite element method to solve problem (2.4). For the analysis, we will restrict our attention to a polygonal domain $\Omega$, which, subsequently, necessarily has reentrant angles. In such a case, the a priori error estimate $(2.10)$ can be improved as follows: $w \in H^{1+r}(\Omega)$ for all $r<\frac{\pi}{\theta}$, where $\theta$ is the largest reentrant angle of $\Omega$, and

$$
\|w\|_{H^{1+r}(\Omega)} \leq C\left[\frac{1}{c^{2}}\|f\|_{L^{2}(\Omega)}^{2}+\sum_{i=1}^{K}\left|\Gamma_{i}\right|\left|\boldsymbol{r}_{i}\right|^{2}\right]^{1 / 2} \leq C\|(f, \boldsymbol{q})\|_{a} .
$$

For the first inequality above, we have used that $\boldsymbol{r}_{i} \cdot \boldsymbol{n}$ is constant on each straight segment $\gamma$ of $\Gamma_{i}$ and, consequently, $\left\|\boldsymbol{r}_{i} \cdot \boldsymbol{n}\right\|_{H^{1 / 2}(\gamma)}^{2}=\left\|\boldsymbol{r}_{i} \cdot \boldsymbol{n}\right\|_{L^{2}(\gamma)}^{2} \leq\left|\boldsymbol{r}_{i}\right|^{2}|\gamma|$. For the second inequality we have used again (2.9).

3. The $h p$ finite element method. In what follows, we introduce an $h p$ finite element method to compute the solutions to problem (2.4). Let $\left\{\mathscr{T}_{h}\right\}$ be a regular family of triangular meshes in $\Omega$. Parameter $h$ stands for the mesh-size; namely, $h:=\max _{T \in \mathscr{T}_{h}} h_{T}$, with $h_{T}$ being the length of the largest edge of the triangle $T$.

We associate with each element $T \in \mathscr{T}_{h}$ a (maximal) polynomial degree $p_{T} \in \mathbb{N}$. We assume that the polynomial degrees of neighboring elements are comparable, i.e., there exists a constant $\gamma>0$ such that

$$
\gamma^{-1} p_{T} \leq p_{T^{\prime}} \leq \gamma p_{T} \quad \forall T, T^{\prime} \in \mathscr{T}_{h} \text { with } T \cap T^{\prime} \neq \emptyset .
$$

We denote by $\boldsymbol{p}:=\left\{p_{T}\right\}_{T \in \mathscr{T}_{h}}$ the family of polynomial degrees for each triangle.

Throughout the paper, we will denote by $C$ a generic positive constant, not necessarily the same at each occurrence, which may depend on the mesh and the degree of the polynomials only through the shape ratio of the triangles and the parameter $\gamma$, respectively.

We define the finite element space as follows:

$$
\mathscr{V}_{h}^{\boldsymbol{p}}:=\mathscr{W}_{h}^{\boldsymbol{p}} \cap L_{0}^{2}(\Omega), \quad \text { with } \quad \mathscr{W}_{h}^{\boldsymbol{p}}:=\left\{v \in H^{1}(\Omega):\left.v\right|_{T} \in \mathscr{P}_{p_{T}} \forall T \in \mathscr{T}_{h}\right\},
$$

where $\mathscr{P}_{p_{T}}$ denotes the set of polynomials of degree at most $p_{T}$. Notice that the definition of $\mathscr{V}_{h}^{p}$ allows for different polynomial degrees on each edge of any triangle. 
Therefore, the space $\left\{\left.v\right|_{T}: v \in \mathscr{V}_{h}^{\boldsymbol{p}}\right\}$ does not necessarily contain all the polynomials of degree $p_{T}$. However, there exists $p_{T}^{\prime} \leq p_{T}$ such that

$$
\mathscr{P}_{p_{T}^{\prime}} \subset\left\{\left.v\right|_{T}: v \in \mathscr{V}_{h}^{\boldsymbol{p}}\right\} \subset \mathscr{P}_{p_{T}} \quad \text { and } \quad p_{T} / p_{T}^{\prime} \leq \gamma,
$$

the latter because of assumption (3.1).

By using these finite elements, we obtain the following discretization of problem (2.4):

Find $\omega_{h}>0$ and a non-vanishing $\left(u_{h}, s_{h}\right) \in \mathscr{V}_{h}^{p} \times \mathbb{R}^{2 K}$ such that

$$
a\left(\left(u_{h}, \boldsymbol{s}_{h}\right),\left(v_{h}, \boldsymbol{t}\right)\right)=\omega_{h}^{2} b\left(\left(u_{h}, \boldsymbol{s}_{h}\right),\left(v_{h}, \boldsymbol{t}\right)\right) \quad \forall\left(v_{h}, \boldsymbol{t}\right) \in \mathscr{V}_{h}^{\boldsymbol{p}} \times \mathbb{R}^{2 K} .
$$

Since the bilinear forms $a$ and $b$ are symmetric and elliptic in $\mathscr{V} \times \mathbb{R}^{2 K}$ and $\mathscr{V}_{h}^{p} \subset \mathscr{V}$, this is a well posed generalized eigenvalue problem with $N+2 K$ positive eigenvalues, where $N:=\operatorname{dim}\left(\mathscr{V}_{h}^{\boldsymbol{p}}\right)$. (See section 6.1 below for a matrix description of this problem.)

Remark 3.1. Since $\mathscr{W}_{h}^{\boldsymbol{p}}=\mathscr{V}_{h}^{\boldsymbol{p}} \oplus \mathbb{R}$, the arguments used in Remark 2.1 allow us to show that (3.3) holds for all test functions $\left(v_{h}, \boldsymbol{t}\right) \in \mathscr{W}_{h}^{\boldsymbol{p}} \times \mathbb{R}^{2 K}$, too.

By testing this equation separately with $\left(v_{h}, \mathbf{0}\right), v_{h} \in \mathscr{V}_{h}$, and $(0, \boldsymbol{t}), \boldsymbol{t} \in \mathbb{R}^{2 K}$, we obtain the following discrete analogues to $(2.2)-(2.3)$, which are actually equivalent to problem (3.3):

$$
\begin{aligned}
\int_{\Omega} \nabla u_{h} \cdot \nabla v_{h} & =\frac{\omega_{h}^{2}}{c^{2}} \int_{\Omega} u_{h} v_{h}+\sum_{i=1}^{K} \boldsymbol{s}_{h i} \cdot\left(\int_{\Gamma_{i}} v_{h} \boldsymbol{n}\right) \quad \forall v_{h} \in \mathscr{V}_{h} . \\
\boldsymbol{s}_{h i} & =\omega_{h}^{2}\left(\frac{\rho_{0}}{k_{i}} \int_{\Gamma_{i}} u_{h} \boldsymbol{n}+\frac{m_{i}}{k_{i}} \boldsymbol{s}_{h i}\right), \quad i=1, \ldots, K .
\end{aligned}
$$

Our first goal is to prove that the solutions of the discrete eigenvalue problem (3.3) converge to those of the spectral problem (2.4). With this aim, we introduce the corresponding solution operator $T_{h}: \mathscr{X} \rightarrow \mathscr{X}$ defined for each $(f, q) \in \mathscr{X}$ by $T_{h}(f, \boldsymbol{q}):=\left(w_{h}, \boldsymbol{r}_{h}\right)$, where $\left(w_{h}, \boldsymbol{r}_{h}\right) \in \mathscr{V}_{h}^{\boldsymbol{p}} \times \mathbb{R}^{2 K}$ is the unique solution of the associated source problem

$$
a\left(\left(w_{h}, \boldsymbol{r}_{h}\right),\left(v_{h}, \boldsymbol{t}\right)\right)=b\left((f, \boldsymbol{q}),\left(v_{h}, \boldsymbol{t}\right)\right) \quad \forall\left(v_{h}, \boldsymbol{t}\right) \in \mathscr{V}_{h}^{\boldsymbol{p}} \times \mathbb{R}^{2 K} .
$$

As in the case of the continuous problem, $\left(w_{h}, \boldsymbol{r}_{h}\right)$ is uniquely determined by the following two equations:

$$
\int_{\Omega} \nabla w_{h} \cdot \nabla v_{h}=\frac{1}{c^{2}} \int_{\Omega} f v_{h}+\sum_{i=1}^{K}\left(\frac{\rho_{0}}{k_{i}} \int_{\Gamma_{i}} f \boldsymbol{n}+\frac{m_{i}}{k_{i}} \boldsymbol{q}_{i}\right) \cdot\left(\int_{\Gamma_{i}} v_{h} \boldsymbol{n}\right) \quad \forall v_{h} \in \mathscr{V}_{h}^{\boldsymbol{p}}
$$

and

$$
\boldsymbol{r}_{h i}=\frac{\rho_{0}}{k_{i}} \int_{\Gamma_{i}} f \boldsymbol{n}+\frac{m_{i}}{k_{i}} \boldsymbol{q}_{i}, \quad i=1, \ldots, K
$$

Once more, by substituting (3.6) into the previous equation, we can write

$$
\int_{\Omega} \nabla w_{h} \cdot \nabla v_{h}=\frac{1}{c^{2}} \int_{\Omega} f v_{h}+\sum_{i=1}^{K} \boldsymbol{r}_{h i} \cdot\left(\int_{\Gamma_{i}} v_{h} \boldsymbol{n}\right) \quad \forall v_{h} \in \mathscr{V}_{h}^{\boldsymbol{p}}
$$


The following result implies the convergence in norm of $T_{h}$ to $T$ as $\max _{T \in \mathscr{T}_{h}} \frac{h_{T}}{p_{T}}$ goes to zero, which will be the main tool to prove spectral convergence.

Lemma 3.2. For all $r<\frac{\pi}{\theta}$, there exists a positive constant $C$ (which depends on $r)$ such that, for all $(f, \boldsymbol{q}) \in \mathscr{X}$,

$$
\left\|T(f, \boldsymbol{q})-T_{h}(f, \boldsymbol{q})\right\|_{a} \leq C\left(\max _{T \in \mathscr{T}_{h}} \frac{h_{T}}{p_{T}}\right)^{r}\|(f, \boldsymbol{q})\|_{a} .
$$

Proof. Let $(f, \boldsymbol{q}) \in \mathscr{X}$. From (2.7) and (3.6) we have that $\boldsymbol{r}_{h}=\boldsymbol{r}$. Hence

$$
\left\|T(f, \boldsymbol{q})-T_{h}(f, \boldsymbol{q})\right\|_{a}=\left|w-w_{h}\right|_{H^{1}(\Omega)} .
$$

Now, from (2.8) and (3.7), Cea's Lemma implies that

$$
\left|w-w_{h}\right|_{H^{1}(\Omega)} \leq\left|w-\left(\Pi_{h}^{p} w-c_{w}\right)\right|_{H^{1}(\Omega)}=\left|w-\Pi_{h}^{p} w\right|_{H^{1}(\Omega)},
$$

where $\Pi_{h}^{p} w$ denotes the $\mathscr{W}_{h}^{p}$-Lagrange interpolant of $w$ (which is well defined because $w \in H^{1+r}(\Omega)$, with $\left.r>0\right)$ and $c_{w}:=\frac{1}{|\Omega|} \int_{\Omega} \Pi_{h}^{p} w$ is the constant such that $\left(\Pi_{h}^{p} w-c_{w}\right) \in \mathscr{V}_{h}^{p}$.

In its turn, by using standard $h p$ error estimates (see, for instance, [3]) we obtain

$$
\left|w-\Pi_{h}^{p} w\right|_{H^{1}(\Omega)}^{2} \leq C \sum_{T \in \mathscr{T}_{h}}\left(\frac{h_{T}}{p_{T}^{\prime}}\right)^{2 r}\|w\|_{H^{1+r}(T)}^{2},
$$

where $p_{T}^{\prime}$ is as defined in (3.2). Thus, the result follows from the above expressions and (2.11).

As a consequence of the classical spectral approximation theory (see [2]) we know that the eigenvalues and eigenspaces of problem (3.3) converge to those of problem (2.4) as $\max _{T \in \mathscr{T}_{h}} \frac{h_{T}}{p_{T}} \rightarrow 0$. From now on, for simplicity, we restrict our attention to a simple eigenvalue $\omega^{2}$ of problem (2.4) with corresponding eigenfunction $(u, s)$ normalized in $\|\cdot\|_{b}$ norm. In such a case, if $\omega^{2}$ is the $j$-th eigenvalue of problem (2.4), then the $j$-th eigenvalue of problem (3.3), which we denote $\omega_{h}^{2}$, is the one which converges to $\omega^{2}$. Moreover, the corresponding eigenfunction $\left(u_{h}, \boldsymbol{s}_{h}\right)$ can be chosen also normalized in $\|\cdot\|_{b}$ norm and such that $\left(u_{h}, \boldsymbol{s}_{h}\right)$ converges to $(u, s)$. In the following theorem we prove a priori error estimates for the approximation of both, the eigenvalue and the eigenfunction.

TheOREM 3.3. For all $r<\frac{\pi}{\theta}$, there exist positive constants $C$ and $\kappa$ such that, if $\max _{T \in \mathscr{T}_{h}} \frac{h_{T}}{p_{T}}<\kappa$, then

$$
\begin{aligned}
\left\|(u, s)-\left(u_{h}, s_{h}\right)\right\|_{a} & \leq C\left(\max _{T \in \mathscr{T}_{h}} \frac{h_{T}}{p_{T}}\right)^{r} \\
\left\|(u, s)-\left(u_{h}, s_{h}\right)\right\|_{b} & \leq C\left(\max _{T \in \mathscr{T}_{h}} \frac{h_{T}}{p_{T}}\right)^{r}\left\|(u, s)-\left(u_{h}, s_{h}\right)\right\|_{a}, \\
\left|\omega^{2}-\omega_{h}^{2}\right| & \leq C\left\|(u, s)-\left(u_{h}, \boldsymbol{s}_{h}\right)\right\|_{a}^{2} .
\end{aligned}
$$

Proof. The estimate (3.8) is a direct consequence of Lemma 3.2 and the classical spectral approximation theory (see [2]). 
To prove (3.9), we resort to a duality argument. Let $P_{h}^{p}$ be the $H^{1}(\Omega)$-elliptic projection onto $\mathscr{V}_{h}^{\boldsymbol{p}}$ defined for any $z \in \mathscr{V}$ by

$$
P_{h}^{p} z \in \mathscr{V}_{h}^{p}: \quad \int_{\Omega} \nabla\left(P_{h}^{p} z-z\right) \cdot \nabla v_{h}=0 \quad \forall v_{h} \in \mathscr{V}_{h}^{p} .
$$

Hence, it is immediate to check that $\left(P_{h}^{p} u, s\right)$ is the orthogonal projection of $(u, s)$ onto $\mathscr{V}_{h}^{\boldsymbol{p}} \times \mathbb{R}^{2 K}$ in the inner product $a(\cdot, \cdot)$; namely,

$$
a\left(\left(P_{h}^{p} u, s\right)-(u, s),\left(v_{h}, \boldsymbol{t}\right)\right)=0 \quad \forall\left(v_{h}, \boldsymbol{t}\right) \in \mathscr{V}_{h}^{\boldsymbol{p}} \times \mathbb{R}^{2 K} .
$$

Consequently, we can apply Lemma 6.4-3 from [13] and we have

$$
\begin{aligned}
\left\|(u, s)-\left(u_{h}, s_{h}\right)\right\|_{b} & \leq C\left\|(u, s)-\left(P_{h}^{p} u, s\right)\right\|_{b} \\
& =C\left[\int_{\Omega} \frac{1}{c^{2}}\left|u-P_{h}^{p} u\right|^{2}+\sum_{i=1}^{K} \frac{\rho_{0}}{k_{i}}\left|\int_{\Gamma_{i}}\left(u-P_{h}^{p} u\right) \boldsymbol{n}\right|^{2}\right]^{1 / 2} .
\end{aligned}
$$

Now, let $\varphi \in \mathscr{V}$ be such that

$$
\int_{\Omega} \nabla \varphi \cdot \nabla \psi=\frac{1}{c^{2}} \int_{\Omega}\left(u-P_{h}^{p} u\right) \psi+\sum_{i=1}^{K} \frac{\rho_{0}}{k_{i}}\left[\int_{\Gamma_{i}}\left(u-P_{h}^{p} u\right) \boldsymbol{n}\right] \cdot\left(\int_{\Gamma_{i}} \psi \boldsymbol{n}\right)
$$

for all $\psi \in \mathscr{V}$. The same arguments leading to the first inequality in (2.11) allow us to conclude that $\varphi \in H^{1+r}(\Omega)$ and

$$
\|\varphi\|_{H^{1+r}(\Omega)} \leq C\left[\frac{1}{c^{2}} \int_{\Omega}\left|u-P_{h}^{p} u\right|^{2}+\sum_{i=1}^{K} \frac{\rho_{0}}{k_{i}}\left|\int_{\Gamma_{i}}\left(u-P_{h}^{p} u\right) \boldsymbol{n}\right|^{2}\right]^{1 / 2} .
$$

Taking $\psi=u-P_{h}^{p} u$ in (3.13) and using (3.11), we obtain

$$
\begin{aligned}
\frac{1}{c^{2}} \| u & -P_{h}^{p} u \|_{L^{2}(\Omega)}^{2}+\sum_{i=1}^{K} \frac{\rho_{0}}{k_{i}}\left|\int_{\Gamma_{i}}\left(u-P_{h}^{p} u\right) \boldsymbol{n}\right|^{2} \\
& =\int_{\Omega} \nabla \varphi \cdot \nabla\left(u-P_{h}^{p} u\right)=\int_{\Omega} \nabla\left[\varphi-\left(\Pi_{h}^{p} \varphi-c_{\varphi}\right)\right] \cdot \nabla\left(u-P_{h}^{p} u\right) \\
& \leq\left|\varphi-\Pi_{h}^{p} \varphi\right|_{H^{1}(\Omega)}\left|u-P_{h}^{p} u\right|_{H^{1}(\Omega)},
\end{aligned}
$$

where, as above, $\Pi_{h}^{p} \varphi$ denotes the $\mathscr{W}_{h}^{p}$-Lagrange interpolant of $\varphi$ and the constant $c_{\varphi}:=\frac{1}{|\Omega|} \int_{\Omega} \Pi_{h}^{p} \varphi$ is such that $\left(\Pi_{h}^{p} \varphi-c_{\varphi}\right) \in \mathscr{V}_{h}^{p}$. Then, using once more standard $h p$ error estimates (cf. [3]), we have

$$
\left|\varphi-\Pi_{h}^{p} \varphi\right|_{H^{1}(\Omega)} \leq C\left(\max _{T \in \mathscr{T}_{h}} \frac{h_{T}}{p_{T}}\right)^{r}\|\varphi\|_{H^{1+r}(\Omega)} .
$$

Thus, (3.9) follows from (3.12), (3.15), (3.16), (3.14), and the inequality

$$
\left|u-P_{h}^{p}(u)\right|_{H^{1}(\Omega)} \leq\left|u-u_{h}\right|_{H^{1}(\Omega)} \leq\left\|(u, s)-\left(u_{h}, s_{h}\right)\right\|_{a},
$$

which in its turn holds because $P_{h}^{p}$ is the $H^{1}(\Omega)$-projector onto $\mathscr{V}_{h}^{p}$. 
Finally (3.10) follows from (3.9) and the well known identity (see, for instance, [2, Lemma 9.1])

$$
\omega_{h}^{2}-\omega^{2}=\left\|\left(u_{h}, \boldsymbol{s}_{h}\right)-(u, s)\right\|_{a}^{2}-\omega^{2}\left\|\left(u_{h}, \boldsymbol{s}_{h}\right)-(u, s)\right\|_{b}^{2} .
$$

Thus we conclude the proof.

Remark 3.4. Actually, instead of (3.9), we have proved that

$$
\left\|(u, s)-\left(u_{h}, s_{h}\right)\right\|_{b} \leq C\left(\max _{T \in \mathscr{T}_{h}} \frac{h_{T}}{p_{T}}\right)^{r}\left|u-u_{h}\right|_{H^{1}(\Omega)} .
$$

This will be used for the a posteriori analysis below.

As a by-product of the second error estimate of Theorem 3.3, in what follows we derive a double order of convergence for the approximation of the velocities of the tubes, which are typically the physical quantities of interest in the applications.

COROLlary 3.5. For all $r<\frac{\pi}{\theta}$, there exist positive constants $C$ and $\kappa$, such that, if $\max _{T \in \mathscr{T}_{h}} \frac{h_{T}}{p_{T}}<\kappa$, then

$$
\left|\boldsymbol{s}_{i}-\boldsymbol{s}_{h i}\right| \leq C\left(\max _{T \in \mathscr{T}_{h}} \frac{h_{T}}{p_{T}}\right)^{r}\left\|(u, s)-\left(u_{h}, s_{h}\right)\right\|_{a}, \quad i=1, \ldots, K .
$$

Proof. Subtracting (3.5) from (2.3), we have

$\boldsymbol{s}_{i}-\boldsymbol{s}_{h i}=\left(\omega^{2}-\omega_{h}^{2}\right)\left(\frac{\rho_{0}}{k_{i}} \int_{\Gamma_{i}} u \boldsymbol{n}+\frac{m_{i}}{k_{i}} \boldsymbol{s}_{i}\right)+\omega_{h}^{2}\left[\frac{\rho_{0}}{k_{i}} \int_{\Gamma_{i}}\left(u-u_{h}\right) \boldsymbol{n}+\frac{m_{i}}{k_{i}}\left(\boldsymbol{s}_{i}-\boldsymbol{s}_{h i}\right)\right]$,

for $i=1, \ldots, K$. To estimate the first term on the right-hand side above we use (3.10), whereas for the second one we use the definition of $\|\cdot\|_{b}$ to write

$$
\left|\frac{\rho_{0}}{k_{i}} \int_{\Gamma_{i}}\left(u-u_{h}\right) \boldsymbol{n}+\frac{m_{i}}{k_{i}}\left(\boldsymbol{s}_{i}-\boldsymbol{s}_{h i}\right)\right| \leq \frac{\rho_{0}^{1 / 2}}{k_{i}^{1 / 2}}\left\|(u, s)-\left(u_{h}, \boldsymbol{s}_{h}\right)\right\|_{b} .
$$

Thus, we conclude the proof by using (3.9).

4. The case of an incompressible fluid. When the fluid is modeled as perfectly incompressible, the first equation in (2.1) has to be substituted by

$$
\Delta u=0 \quad \text { in } \Omega .
$$

This equation can be seen as the limit of the corresponding one in (2.1) as the sound speed $c$ goes to infinity. In such a case, most of the analysis of the previous sections remains valid. In fact, in general, it is enough to delete all the terms multiplied by $\frac{1}{c^{2}}$. In what follows we will discuss those parts of the analysis that must be changed for an incompressible fluid.

First, the condition $\int_{\Omega} u=0$ is lost for an incompressible fluid. Indeed, in this case, the velocity potential $u$ is determined only up to an additive constant. Therefore, we can still enforce the zero-mean constraint and, consequently, we can use the spaces $L_{0}^{2}(\Omega)$ and $\mathscr{V}:=H^{1}(\Omega) \cap L_{0}^{2}(\Omega)$ for the analysis.

Secondly, the bilinear form

$$
b((u, s),(v, \boldsymbol{t})):=\sum_{i=1}^{K} \frac{\rho_{0}}{k_{i}}\left(\int_{\Gamma_{i}} u \boldsymbol{n}+\frac{m_{i}}{\rho_{0}} \boldsymbol{s}_{i}\right) \cdot\left(\int_{\Gamma_{i}} v \boldsymbol{n}+\frac{m_{i}}{\rho_{0}} \boldsymbol{t}_{i}\right),
$$


which results from dropping out the term multiplied by $\frac{1}{c^{2}}$ in (2.6), is no longer elliptic on $\mathscr{X}$. Consequently, $\|\cdot\|_{b}$ is a seminorm rather than a norm in this case.

This fact does not affect the spectral characterization of $T$, since, anyway, the operator is still compact and self-adjoint with respect to the inner product $a(\cdot, \cdot)$. What changes significantly is the distribution of its spectrum. In fact, now $T$ is an operator with finite rank $2 K$, as can be easily seen from the equations analogous to (2.7) and (2.8); namely, $T(f, \boldsymbol{q})=(w, \boldsymbol{r})$, with

$$
\boldsymbol{r}_{i}=\frac{\rho_{0}}{k_{i}} \int_{\Gamma_{i}} f \boldsymbol{n}+\frac{m_{i}}{k_{i}} \boldsymbol{q}_{i}, \quad i=1, \ldots, K,
$$

and

$$
\int_{\Omega} \nabla w \cdot \nabla v=\sum_{i=1}^{K} \boldsymbol{r}_{i} \cdot\left(\int_{\Gamma_{i}} v \boldsymbol{n}\right) \quad \forall v \in \mathscr{V}
$$

Therefore, 0 is an infinite-multiplicity eigenvalue of $T$ and the rest of the spectrum consists of $2 K$ positive eigenvalues (counted according to their respective multiplicities).

The discretization of the corresponding problem follows the same lines as that for a compressible fluid. The analysis is also much the same and Lemma 3.2, Theorem 3.3 and Corollary 3.5 all hold true. The only difference in the proofs arise in that of the estimate (3.9) from Theorem 3.3. In fact, since for an incompressible fluid the bilinear form $b$ is not elliptic on $\mathscr{X}$, we cannot use Lemma 6.4-3 from [13] to prove the first inequality from (3.12). We avoid this drawback by using a variant of that lemma valid for non negative bilinear forms, which was proved in [1] (cf. Lemma 3.2 therein). The rest of the proof runs identically.

On the other hand, let us remark that, in most cases, all the inequalities from the previous sections hold with constants $C$ that are independent of the sound speed $c$, as far as $c \in\left[c_{0},+\infty\right)$, with $c_{0}>0$. The only possible exception happens only in an extremely rare case, that we discuss in what follows.

Let us recall first something that was shown in [4]: the lowest $2 K$ vibration frequencies of the problem with a compressible fluid converge to those of the problem with an incompressible fluid, as the sound speed $c$ goes to infinity (all the other vibration frequencies in the case of a compressible fluid diverge to $+\infty$ ).

On the other hand, the constants in the estimates of Theorem 3.3 depend on the distance of the eigenvalue $\frac{1}{\omega^{2}}$ of $T$ to the rest of the spectrum of this operator (see $[2,13])$. In the case that a multiple eigenvalue of the operator for an incompressible fluid were the limit of several simple eigenvalues of the corresponding operator for a compressible fluid, this distance would go to zero as $c \rightarrow+\infty$. Thus, in principle, in such a case, the constants in the estimates of Theorem 3.3 could blow up as $c$ goes to infinity.

However, typically, multiple eigenvalues occur only because of some symmetry of the domain. In such a case the multiplicities of the operators corresponding to compressible and incompressible fluids are usually the same. In principle, Theorem 3.3 does not apply to this case, since it does not cover multiple eigenvalues. However, in practice, it is standard to take advantage of that symmetry to reformulate the problem in a reduced domain in which the eigenvalue to be computed is simple. This is what we have actually done in the numerical experiments reported in section 7 below.

Anyway, for the analogous to Theorem 3.3 to hold with constants independent of the sound speed, we must add a hypothesis to this theorem (and subsequently to 
Corollary 3.5, since it follows from this theorem) to rule out the possibility of simple eigenvalues of the compressible problem converging to a multiple eigenvalue of the incompressible one. Thus, we are lead to the following result:

THEOREM 4.1. Let $\omega^{2}$ be a simple eigenvalue of problem (2.4) with $b$ defined by (2.6) (compressible fluid), which converges to a simple eigenvalue of the same problem but with $b$ defined by (4.1) (incompressible fluid) as $c \rightarrow+\infty$. For all $r<\frac{\pi}{\theta}$, there exist positive constants $C, \kappa$, and $c_{0}$, such that, if $\max _{T \in \mathscr{T}_{h}} \frac{h_{T}}{p_{T}}<\kappa$ and $c \geq c_{o}$, then

$$
\begin{aligned}
\left\|(u, s)-\left(u_{h}, s_{h}\right)\right\|_{a} & \leq C\left(\max _{T \in \mathscr{T}_{h}} \frac{h_{T}}{p_{T}}\right)^{r}, \\
\left\|(u, s)-\left(u_{h}, s_{h}\right)\right\|_{b} & \leq C\left(\max _{T \in \mathscr{T}_{h}} \frac{h_{T}}{p_{T}}\right)^{r}\left\|(u, s)-\left(u_{h}, s_{h}\right)\right\|_{a}, \\
\left|\omega^{2}-\omega_{h}^{2}\right| & \leq C\left\|(u, s)-\left(u_{h}, s_{h}\right)\right\|_{a}^{2}, \\
\left|s_{i}-s_{h i}\right| & \leq C\left(\max _{T \in \mathscr{T}_{h}} \frac{h_{T}}{p_{T}}\right)^{r}\left\|(u, s)-\left(u_{h}, s_{h}\right)\right\|_{a}, \quad i=1, \ldots, K,
\end{aligned}
$$

all the estimates with constants $C$ independent of $c$.

The relevance of this theorem is that it shows that the proposed $h p$ finite element method can be safely used for nearly incompressible fluids (i.e., $c$ very large), since the error estimates do not deteriorate as the sound speed $c$ becomes larger.

5. A posteriori error estimator. For an efficient use of an $h p$ finite element method, an adaptive scheme is typically needed. Such a scheme is based on a posteriori error indicators and a strategy to decide at each step whether to perform $h$ - or $p$ refinement. In this section we derive a residual type a posteriori error estimator and prove that it is equivalent to the error of the computed eigenfunction up to higher order terms.

The aim of the adaptive scheme is to obtain meshes more refined around the singular points of the eigenfunction, accordingly to the strength of the respective singularities. As will be shown in the numerical test of section 7 below, different eigenfunctions do not necessarily have the same singularity strength at the same points, and this happens even for linearly independent eigenfunctions of a same multiple eigenvalue. Since for multiple eigenvalues, in general, it is not possible to single out numerical approximations of a particular eigenfunction, the adaptive scheme we are going to introduce will be useful only for simple eigenvalues. However, we show in section 7.1 below how it can be applied to multiple eigenvalues arising from the symmetry of the problem, which is the most usual case in practice.

Let $(\omega, u, s)$ and $\left(\omega_{h}, u_{h}, \boldsymbol{s}_{h}\right)$ be solutions of problems (2.4) and (3.3), respectively. We assume that $\omega^{2}$ is a simple eigenvalue of problem (2.4), that $\omega_{h}^{2}$ is the (also simple) eigenvalue of problem (3.3) which converges to $\omega^{2}$ as $\max _{T \in \mathscr{T}_{h}} \frac{h_{T}}{p_{T}} \rightarrow 0$, and that $(u, s)$ and $\left(u_{h}, s_{h}\right)$ are chosen normalized in norm $\|\cdot\|_{b}$ and such that the estimates from Theorem 3.3 and Corollary 3.5 hold true.

We denote by $e_{u}:=u-u_{h}$ and $\boldsymbol{e}_{\boldsymbol{s}}:=\boldsymbol{s}-\boldsymbol{s}_{h}$ the respective errors. We will derive an a posteriori estimator of these errors measured in $\|\cdot\|_{a}$. The main reason for this choice is that this is the natural (energy) norm of this problem, which makes the computations that follow simpler. Moreover, controlling this norm,

$$
\left\|\left(e_{u}, e_{\boldsymbol{s}}\right)\right\|_{a}=\left[\left\|\nabla u-\nabla u_{h}\right\|_{L^{2}(\Omega)}^{2}+\sum_{i=1}^{K} \frac{m_{i}}{\rho_{0}}\left|\boldsymbol{s}_{i}-\boldsymbol{s}_{h i}\right|^{2}\right]^{1 / 2},
$$


allows keeping control on the two quantities that are typically of interest in applications: the computed fluid velocity amplitude, $\nabla u_{h}$, and the tubes velocity amplitudes, $\boldsymbol{s}_{h i}$. However, let us remark that, according to Corollary 3.5, the latter are of higher order than the former, so that the most relevant error measure is $\left\|\nabla e_{u}\right\|_{L^{2}(\Omega)}$.

We introduce some notation that will be used in the definition and analysis of the error estimator. For any $T \in \mathscr{T}_{h}$ let $\mathscr{E}_{T}$ denote the set of edges of $T$ and $\mathscr{E}:=$ $\bigcup_{T \in \mathscr{T}_{h}} \mathscr{E}_{T}$. We decompose $\mathscr{E}$ in disjoint sets $\mathscr{E}_{\Gamma_{i}}:=\left\{\ell \in \mathscr{E}: \ell \subset \Gamma_{i}\right\}, 0 \leq i \leq K$, and $\mathscr{E}_{\Omega}:=\mathscr{E} \backslash \bigcup_{i=0}^{K} \mathscr{E}_{\Gamma_{i}}$.

For each $\ell \in \mathscr{E}_{\Omega}$ we choose a unit normal vector $\boldsymbol{n}_{\ell}$ and denote the two triangles sharing this edge $T_{\text {in }}$ and $T_{\text {out }}$, with $\boldsymbol{n}_{\ell}$ pointing outwards $T_{\text {in }}$. For $v_{h} \in \mathscr{V}_{h}$ we set

$$
\llbracket \frac{\partial v_{h}}{\partial n} \rrbracket_{\ell}:=\nabla\left(\left.v_{h}\right|_{T_{\text {out }}}\right) \cdot \boldsymbol{n}_{\ell}-\nabla\left(\left.v_{h}\right|_{T_{\text {in }}}\right) \cdot \boldsymbol{n}_{\ell},
$$

which corresponds to the jump of the normal derivative of $v_{h}$ across the edge $\ell$. Note that this value is independent of the chosen direction of the normal vector $\boldsymbol{n}_{\ell}$.

The first step for the a posteriori error analysis in spectral problems is to write down a property which plays the role of Galerkin orthogonality in source problems. With this aim, we use equations (2.4) and (3.3) with a discrete test function $\left(v_{h}, \boldsymbol{t}\right) \in$ $\mathscr{W}_{h}^{\boldsymbol{p}} \times \mathbb{R}^{2 K}$ (notice that this can be done because of Remarks 2.1 and 3.1). Thus, we obtain

$$
a\left(\left(e_{u}, \boldsymbol{e}_{\boldsymbol{s}}\right),\left(v_{h}, \boldsymbol{t}\right)\right)=\omega^{2} b\left((u, \boldsymbol{s}),\left(v_{h}, \boldsymbol{t}\right)\right)-\omega_{h}^{2} b\left(\left(u_{h}, \boldsymbol{s}_{h}\right),\left(v_{h}, \boldsymbol{t}\right)\right) .
$$

On the other hand, for any test function $(v, t) \in \mathscr{V} \times \mathbb{R}^{2 K}$, from (2.4) we have

$$
\begin{aligned}
a\left(\left(e_{u}, \boldsymbol{e}_{\boldsymbol{s}}\right),(v, \boldsymbol{t})\right)= & {\left[\omega^{2} b((u, \boldsymbol{s}),(v, \boldsymbol{t}))-\omega_{h}^{2} b\left(\left(u_{h}, \boldsymbol{s}_{h}\right),(v, \boldsymbol{t})\right)\right] } \\
& +\left[\omega_{h}^{2} b\left(\left(u_{h}, \boldsymbol{s}_{h}\right),(v, \boldsymbol{t})\right)-a\left(\left(u_{h}, \boldsymbol{s}_{h}\right),(v, \boldsymbol{t})\right)\right] .
\end{aligned}
$$

As will be shown below, the first expression between brackets will lead to a higher order term. So, we focus on the second one. This vanishes for $(0, \boldsymbol{t}) \in \mathscr{V}_{h}^{\boldsymbol{p}} \times \mathbb{R}^{2 K}$ because of (3.3). Thus, we have

$$
\begin{aligned}
& \omega_{h}^{2} b\left(\left(u_{h}, \boldsymbol{s}_{h}\right),(v, \boldsymbol{t})\right)-a\left(\left(u_{h}, \boldsymbol{s}_{h}\right),(v, \boldsymbol{t})\right) \\
&= \omega_{h}^{2} b\left(\left(u_{h}, \boldsymbol{s}_{h}\right),(v, \mathbf{0})\right)-a\left(\left(u_{h}, \boldsymbol{s}_{h}\right),(v, \mathbf{0})\right) \\
&= \sum_{T \in \mathscr{T}_{h}} \int_{T}\left(\Delta u_{h}+\frac{\omega_{h}^{2}}{c^{2}} u_{h}\right) v+\frac{1}{2} \sum_{\ell \in \mathscr{E}_{\Omega}} \int_{\ell} \llbracket \frac{\partial u_{h}}{\partial n} \rrbracket_{\ell} v \\
&-\sum_{\ell \in \mathscr{E}_{\Gamma_{0}}} \int_{\ell} \frac{\partial u_{h}}{\partial n} v-\sum_{i=1}^{K} \sum_{\ell \in \mathscr{E}_{\Gamma_{i}}} \int_{\ell}\left(\frac{\partial u_{h}}{\partial n}-\boldsymbol{s}_{h i} \cdot \boldsymbol{n}\right) v,
\end{aligned}
$$

where we have used (2.6), integration by parts, and (3.5). The right-hand side above can be written in terms of residuals of problem (2.1). In fact, for each $\ell \in \mathscr{E}$, we define the edge residual

$$
J_{\ell}:= \begin{cases}\frac{1}{2} \llbracket \frac{\partial u_{h}}{\partial n} \|_{\ell}, & \text { if } \ell \in \mathscr{E}_{\Omega}, \\ -\frac{\partial u_{h}}{\partial n}, & \text { if } \ell \in \mathscr{E}_{\Gamma_{0}}, \\ -\left(\frac{\partial u_{h}}{\partial n}-\boldsymbol{s}_{h i} \cdot \boldsymbol{n}\right), & \text { if } \ell \in \mathscr{E}_{\Gamma_{i}}, \quad i=1, \ldots, K,\end{cases}
$$


and, for each $T \in \mathscr{T}_{h}$, the volumetric residual

$$
R_{T}:=\Delta\left(\left.u_{h}\right|_{T}\right)+\left.\frac{\omega_{h}^{2}}{c^{2}} u_{h}\right|_{T}
$$

Using these residuals in (5.2), we obtain the error equation of the problem:

$$
\begin{aligned}
a\left(\left(e_{u}, \boldsymbol{e}_{\boldsymbol{s}}\right),(v, \boldsymbol{t})\right)= & {\left[\omega^{2} b((u, \boldsymbol{s}),(v, \boldsymbol{t}))-\omega_{h}^{2} b\left(\left(u_{h}, \boldsymbol{s}_{h}\right),(v, \boldsymbol{t})\right)\right] } \\
& +\sum_{T \in \mathscr{T}_{h}}\left(\int_{T} R_{T} v+\sum_{\ell \in \mathscr{E}_{T}} \int_{\ell} J_{\ell} v\right) \quad \forall(v, \boldsymbol{t}) \in \mathscr{V} \times \mathbb{R}^{2 K} .
\end{aligned}
$$

Next step will be to apply this equation to $(v, \mathbf{0})$, with $v=e_{u}-I_{h}^{p} e_{u}$, where $I_{h}^{p}$ denotes an $h p$ Clément interpolation operator in $\mathscr{W}_{h}^{\boldsymbol{p}}$, which was defined in [9]. The following error estimate, which can be easily proved by using the results from this reference, hold for this interpolation:

$$
\sum_{T \in \mathscr{T}_{h}}\left[\frac{p_{T}^{2}}{h_{T}^{2}}\left\|e_{u}-I_{h}^{p} e_{u}\right\|_{L^{2}(T)}^{2}+\sum_{\ell \in \mathscr{E}_{T}} \frac{p_{\ell}}{|\ell|}\left\|e_{u}-I_{h}^{p} e_{u}\right\|_{L^{2}(\ell)}^{2}\right] \leq C\left|e_{u}\right|_{H^{1}(\Omega)}^{2},
$$

where $p_{\ell}:=\max \left\{p_{T}: \mathscr{E}_{T} \ni \ell\right\}$.

Taking $(v, \boldsymbol{t})=\left(e_{u}-I_{h}^{p} e_{u}, \mathbf{0}\right)$ as test function in (5.3) and using (5.1) with $\left(v_{h}, \boldsymbol{t}\right)=$ $\left(I_{h}^{p} e_{u}, e_{s}\right)$, some algebraic manipulations allow us to write

$$
\begin{aligned}
\left\|\left(e_{u}, \boldsymbol{e}_{\boldsymbol{s}}\right)\right\|_{a}^{2}= & a\left(\left(e_{u}, \boldsymbol{e}_{\boldsymbol{s}}\right)\left(e_{u}-I_{h}^{p} e_{u}, \mathbf{0}\right)\right)+a\left(\left(e_{u}, \boldsymbol{e}_{\boldsymbol{s}}\right),\left(I_{h}^{p} e_{u}, \boldsymbol{e}_{\boldsymbol{s}}\right)\right) \\
= & {\left[\omega^{2} b\left((u, \boldsymbol{s}),\left(e_{u}, \boldsymbol{e}_{\boldsymbol{s}}\right)\right)-\omega_{h}^{2} b\left(\left(u_{h}, \boldsymbol{s}_{h}\right),\left(e_{u}, \boldsymbol{e}_{\boldsymbol{s}}\right)\right)\right] } \\
& +\sum_{T \in \mathscr{T}_{h}}\left[\int_{T} R_{T}\left(e_{u}-I_{h}^{p} e_{u}\right)+\sum_{\ell \in \mathscr{E}_{T}} \int_{\ell} J_{\ell}\left(e_{u}-I_{h}^{p} e_{u}\right)\right] .
\end{aligned}
$$

To estimate the first term on the right-hand side above, we recall that $\|(u, s)\|_{b}=$ $\left\|\left(u_{h}, s_{h}\right)\right\|_{b}=1$, to write

$$
\begin{aligned}
{\left[\omega^{2} b((u, s)\right.} & \left.\left.,\left(e_{u}, \boldsymbol{e}_{\boldsymbol{s}}\right)\right)-\omega_{h}^{2} b\left(\left(u_{h}, \boldsymbol{s}_{h}\right),\left(e_{u}, \boldsymbol{e}_{\boldsymbol{s}}\right)\right)\right] \\
& =\omega^{2}\|(u, s)\|_{b}^{2}-\left(\omega^{2}+\omega_{h}^{2}\right) b\left((u, s),\left(u_{h}, \boldsymbol{s}_{h}\right)\right)+\omega_{h}^{2}\left\|\left(u_{h}, \boldsymbol{s}_{h}\right)\right\|_{b}^{2} \\
& =\left(\omega^{2}+\omega_{h}^{2}\right)-\left(\omega^{2}+\omega_{h}^{2}\right) b\left((u, s),\left(u_{h}, \boldsymbol{s}_{h}\right)\right) \\
& =\frac{\omega^{2}+\omega_{h}^{2}}{2}\left\|(u, s)-\left(u_{h}, \boldsymbol{s}_{h}\right)\right\|_{b}^{2} \leq C\left(\max _{T \in \mathscr{T}_{h}} \frac{h_{T}}{p_{T}}\right)^{2 r}\left|e_{u}\right|_{H^{1}(\Omega)}^{2},
\end{aligned}
$$

where we have used Remark 3.4 for the last inequality. Notice that, since $\left|e_{u}\right|_{H^{1}(\Omega)} \leq$ $\left\|\left(e_{u}, \boldsymbol{e}_{\boldsymbol{s}}\right)\right\|_{a}$, this shows that this is a higher order term in (5.5).

To estimate the second term on the right-hand side of (5.5), we use CauchySchwarz inequality and (5.4) to write

$$
\begin{aligned}
\sum_{T \in \mathscr{T}_{h}}\left[\int_{T} R_{T}\left(e_{u}-I_{h}^{p} e_{u}\right)+\sum_{\ell \in \mathscr{E}_{T}} \int_{\ell} J_{\ell}\left(e_{u}-I_{h}^{p} e_{u}\right)\right] \\
\leq C\left\{\sum_{T \in \mathscr{T}_{h}}\left[\frac{h_{T}^{2}}{p_{T}^{2}}\left\|R_{T}\right\|_{L^{2}(T)}^{2}+\sum_{\ell \in \mathscr{E}_{T}} \frac{|\ell|}{p_{\ell}}\left\|J_{\ell}\right\|_{L^{2}(\ell)}^{2}\right]\right\}^{1 / 2}\left|e_{u}\right|_{H^{1}(\Omega)}
\end{aligned}
$$


This suggests us to define the local error indicators

$$
\eta_{T}^{2}:=\frac{h_{T}^{2}}{p_{T}^{2}}\left\|R_{T}\right\|_{L^{2}(T)}^{2}+\sum_{\ell \in \mathscr{E}_{T}} \frac{|\ell|}{p_{\ell}}\left\|J_{\ell}\right\|_{L^{2}(\ell)}^{2}, \quad T \in \mathscr{T}_{h},
$$

(recall that $p_{\ell}:=\max \left\{p_{T}: \mathscr{E}_{T} \ni \ell\right\}$ ) and the corresponding global error estimator

$$
\eta_{\Omega}^{2}:=\sum_{T \in \mathscr{T}_{h}} \eta_{T}^{2}
$$

Thus, from (5.5)-(5.9), we conclude the following result.

TheOREM 5.1. There exists a positive constant $C$ such that

$$
\left\|\left(e_{u}, \boldsymbol{e}_{\boldsymbol{s}}\right)\right\|_{a} \leq C\left[\eta_{\Omega}+\left(\max _{T \in \mathscr{T}_{h}} \frac{h_{T}}{p_{T}}\right)^{2 r}\left|e_{u}\right|_{H^{1}(\Omega)}\right] .
$$

Since the term $\left(\max _{T \in \mathscr{T}_{h}} \frac{h_{T}}{p_{T}}\right)^{2 r}\left|e_{u}\right|_{H^{1}(\Omega)}$ is clearly asymptotically negligible with respect to the left-hand side of this estimate, we conclude that the error of the method $\left\|\left(e_{u}, \boldsymbol{e}_{\boldsymbol{s}}\right)\right\|_{a}$ is bounded above by the global estimator $\eta_{\Omega}$ up to a higher order term and a multiplicative constant. In other words, $\eta_{\Omega}$ is an asymptotically reliable a posteriori error estimate.

In order to guarantee that the error indicator is efficient to guide an adaptive refinement scheme, in what follows we will prove that $\eta_{T}$ is bounded by the $H^{1}$-norm of the error in a neighborhood of $T$, up to some additional terms which will be shown to be (globally) asymptotically negligible.

With this end, the first step is to prove upper estimates for all the residuals appearing in the definition of the error indicators $\eta_{T}$ (cf. (5.8)). The following two lemmas provide such estimates for the volumetric and edge residuals, respectively.

Lemma 5.2. There exists a positive constant $C$ such that

$$
\frac{h_{T}}{p_{T}}\left\|R_{T}\right\|_{L^{2}(T)} \leq C p_{T}\left|e_{u}\right|_{H^{1}(T)}+\frac{h_{T}}{c^{2}}\left\|\omega^{2} u-\omega_{h}^{2} u_{h}\right\|_{L^{2}(T)} .
$$

Lemma 5.3. For all $\delta>0$, there exists a positive constant $C_{\delta}$ such that, if $\ell \in \mathscr{E}_{\Omega} \cup \mathscr{E}_{\Gamma_{0}}$, then

$$
\frac{|\ell|^{1 / 2}}{p_{\ell}^{1 / 2}}\left\|J_{\ell}\right\|_{L^{2}(\ell)} \leq C_{\delta}\left[p_{\ell}^{1+\delta}\left|e_{u}\right|_{H^{1}\left(\omega_{\ell}\right)}+\frac{p_{\ell}^{\delta}|\ell|}{c^{2}}\left\|\omega^{2} u-\omega_{h}^{2} u_{h}\right\|_{L^{2}\left(\omega_{\ell}\right)}\right]
$$

and, if $\ell \in \mathscr{E}_{\Gamma_{i}}, 1 \leq i \leq K$, then

$$
\frac{|\ell|^{1 / 2}}{p_{\ell}^{1 / 2}}\left\|J_{\ell}\right\|_{L^{2}(\ell)} \leq C_{\delta}\left[p_{\ell}^{1+\delta}\left|e_{u}\right|_{H^{1}\left(\omega_{\ell}\right)}+\frac{p_{\ell}^{\delta}|\ell|}{c^{2}}\left\|\omega^{2} u-\omega_{h}^{2} u_{h}\right\|_{L^{2}\left(\omega_{\ell}\right)}+p_{\ell}^{\delta}|\ell|\left|s_{i}-s_{h i}\right|\right],
$$

where $\omega_{\ell}:=\bigcup\left\{T \in \mathscr{T}_{h}: \ell \in \mathscr{E}_{T}\right\}$.

We do not include the proofs of these two lemmas since they are similar to those of Lemmas 4.1 and 4.2 from [1], respectively. They are based on using the error equation (5.3) with test functions of the form $(v, \mathbf{0})$, for different convenient bubble functions $v$.

Now we are in a position to prove the following upper estimate for $\eta_{T}$. 
TheOREM 5.4. For all $\delta>0$, there exists a positive constant $C_{\delta}$ such that for all $T \in \mathscr{T}_{h}$, if $T$ has only inner edges (i.e., edges $\ell \in \mathscr{E}_{\Omega}$ ), then

$$
\eta_{T} \leq C_{\delta} p_{T}^{1+\delta}\left[\left|e_{u}\right|_{H^{1}\left(\omega_{T}\right)}+\frac{h_{T}}{p_{T} c^{2}}\left\|\omega^{2} u-\omega_{h}^{2} u_{h}\right\|_{L^{2}\left(\omega_{T}\right)}\right]
$$

and, if $T$ has an edge lying on $\Gamma_{i}, i=1, \ldots, K$, then

$$
\eta_{T} \leq C_{\delta} p_{T}^{1+\delta}\left[\left|e_{u}\right|_{H^{1}\left(\omega_{T}\right)}+\frac{h_{T}}{p_{T} c^{2}}\left\|\omega^{2} u-\omega_{h}^{2} u_{h}\right\|_{L^{2}\left(\omega_{T}\right)}+\frac{h_{T}}{p_{T}}\left|s_{i}-s_{h i}\right|\right],
$$

where $\omega_{T}:=\bigcup\left\{T^{\prime}: T\right.$ and $T^{\prime}$ share an edge $\}$.

Proof. It is an immediate consequence of Lemmas 5.2 and 5.3 and the assumption (3.1).

The estimates of this theorem have two drawbacks. The first one is that the 'constants' $C_{\delta} p_{T}^{1+\delta}$ obviously depend on the polynomial degree $p_{T}$. The second one is that the right-hand sides include additional terms $\frac{h_{T}}{p_{T} c^{2}}\left\|\omega^{2} u-\omega_{h}^{2} u_{h}\right\|_{L^{2}\left(\omega_{T}\right)}$ and $\frac{h_{T}}{p_{T}}\left|\boldsymbol{s}_{i}-\boldsymbol{s}_{h i}\right|$ that should be negligible for (5.10) and (5.11) to be actual efficiency estimates. We will address the first drawback at the end of this section. Regarding the second one, what we can prove is that the global estimator $\eta_{\Omega}$ defined from $\eta_{T}$ (cf. (5.9)) satisfies an efficiency estimate up to higher order terms. In fact, from Lemmas 5.2 and 5.3 , for all $\delta>0$ we have the following global lower error estimate:

$$
\eta_{\Omega} \leq C_{\delta}(\max \boldsymbol{p})^{1+\delta}\left(\left\|\left(e_{u}, \boldsymbol{e}_{\boldsymbol{s}}\right)\right\|_{a}^{2}+\text { h.o.t. }\right)^{1 / 2},
$$

where

$$
\begin{aligned}
\text { h.o.t. }:= & \sum_{T \in \mathscr{T}_{h}} \frac{h_{T}^{2}}{p_{T}^{2} c^{2}}\left\|\omega^{2} u-\omega_{h}^{2} u_{h}\right\|_{L^{2}(T)}^{2}+\sum_{i=1}^{K} \sum_{\ell \in \mathscr{E}_{\Gamma_{i}}} \frac{|\ell|^{2}}{p_{\ell}^{2}}\left|s_{i}-\boldsymbol{s}_{i h}\right|^{2} \\
\leq & \left(\max _{T \in \mathscr{T}_{h}} \frac{h_{T}}{p_{T}}\right)^{2}\left[\frac{\omega^{2}}{c^{2}}\left\|u-u_{h}\right\|_{L^{2}(\Omega)}^{2}+\frac{\left|\omega^{2}-\omega_{h}^{2}\right|}{c^{2}}\left\|u_{h}\right\|_{L^{2}(\Omega)}^{2}\right] \\
& +\frac{2}{\min p}\left(\max _{T \in \mathscr{T}_{h}} \frac{h_{T}}{p_{T}}\right) \sum_{i=1}^{K}\left|\Gamma_{i}\right|\left|\boldsymbol{s}_{i}-\boldsymbol{s}_{i h}\right|^{2} .
\end{aligned}
$$

Hence, from (3.9), (3.10), and the fact that $\left\|\left(u_{h}, s_{h}\right)\right\|_{b}=1$, we have

$$
\begin{aligned}
\text { h.o.t. } \leq C\left[\left(\max _{T \in \mathscr{T}_{h}} \frac{h_{T}}{p_{T}}\right)^{2+2 r}\left\|\left(e_{u}, \boldsymbol{e}_{\boldsymbol{s}}\right)\right\|_{a}^{2}+\left(\max _{T \in \mathscr{T}_{h}} \frac{h_{T}}{p_{T}}\right)^{2}\left\|\left(e_{u}, \boldsymbol{e}_{\boldsymbol{s}}\right)\right\|_{a}^{4}\right. \\
\left.+\left(\max _{T \in \mathscr{T}_{h}} \frac{h_{T}}{p_{T}}\right)^{1+2 r}\left\|\left(e_{u}, \boldsymbol{e}_{\boldsymbol{s}}\right)\right\|_{a}^{2}\right],
\end{aligned}
$$

which is clearly a higher order term as compared with $\left\|\left(e_{u}, \boldsymbol{e}_{\boldsymbol{s}}\right)\right\|_{a}^{2}$.

On the other hand, as mentioned above, the efficiency estimate (5.12) is suboptimal in that the equivalence constant depends on the polynomial degree. In contrast to the case of $h$-refinement, it seems to be an open question whether uniform reliability and efficiency can be achieved for an $h p$ a posteriori estimator. In fact, to the best of the authors' knowledge, proofs of upper and lower bounds for the energy norm of the 
error, both independent of the polynomial degree $p$, have not been reported yet for any $h p$ finite element method. Nevertheless, according to the experiments reported in section 7 , this seems to be just a theoretical issue. Indeed, the degrees achieved in the experiments are not that large, so that the factor $p^{1+\delta}$ can be considered bounded for practical purposes.

6. Implementation issues. In this section we analyze some aspects concerning the computer solution of the discrete problem. In particular, we give a matrix description of the algebraic eigenvalue problem to be solved and an adaptive refinement strategy based on the error indicators $\eta_{T}$.

6.1. The matrix eigenvalue problem. To write problem (3.3) in matrix form, we introduce the finite element nodal basis $\left\{\varphi_{h, i}\right\}_{i=1}^{N}$ of $\mathscr{W}_{h}^{\boldsymbol{p}}$ corresponding to the set of nodes $\mathscr{N}:=\left\{P_{i}\right\}_{i=1}^{N}$ of this space (i.e., $\left.\varphi_{h, i}\left(P_{j}\right)=\delta_{i j}, i, j=1, \ldots, N\right)$. Let $\boldsymbol{u}_{h}:=\left(u_{h}\left(P_{i}\right)\right) \in \mathbb{R}^{N}$ be the vector of nodal components. Then, (3.3) leads to

$$
\underbrace{\left(\begin{array}{cc}
\boldsymbol{K}_{\Delta} & \mathbf{0} \\
\mathbf{0} & \boldsymbol{K}_{22}
\end{array}\right)}_{\mathcal{K}}\left(\begin{array}{l}
\boldsymbol{u}_{h} \\
\boldsymbol{s}_{h}
\end{array}\right)=\omega_{h}^{2} \underbrace{\left(\begin{array}{cc}
\frac{1}{c^{2}} \boldsymbol{M}_{\Delta}+\boldsymbol{M}_{\Gamma} & \boldsymbol{M}_{12} \\
\boldsymbol{M}_{12}^{\mathrm{t}} & \boldsymbol{M}_{22}
\end{array}\right)}_{\mathcal{M}}\left(\begin{array}{c}
\boldsymbol{u}_{h} \\
\boldsymbol{s}_{h}
\end{array}\right),
$$

where $\boldsymbol{s}_{h} \in \mathbb{R}^{2 K}$ is as in (3.3),

$$
\boldsymbol{K}_{\Delta}:=\left(\int_{\Omega} \nabla \varphi_{h, i} \cdot \nabla \varphi_{h, j}\right) \quad \text { and } \quad \boldsymbol{M}_{\Delta}:=\left(\int_{\Omega} \varphi_{h, i} \varphi_{h, j}\right) \in \mathbb{R}^{N \times N}
$$

are the standard stiffness and mass matrices, respectively, of the Laplace operator, and $\boldsymbol{K}_{22}, \boldsymbol{M}_{22} \in \mathbb{R}^{2 K \times 2 K}$ are the diagonal matrices defined as follows:

$\boldsymbol{K}_{22}:=\frac{1}{\rho_{0}} \operatorname{diag}\left(m_{1}, m_{1}, \ldots, m_{K}, m_{K}\right), \boldsymbol{M}_{22}:=\frac{1}{\rho_{0}} \operatorname{diag}\left(\frac{m_{1}^{2}}{k_{1}}, \frac{m_{1}^{2}}{k_{1}}, \cdots, \frac{m_{K}^{2}}{k_{K}}, \frac{m_{K}^{2}}{k_{K}}\right)$.

To define $\boldsymbol{M}_{\Gamma}$ and $\boldsymbol{M}_{12}$, we previously introduce the auxiliary matrices

$$
\boldsymbol{B}_{j}:=\left(\begin{array}{lll}
\int_{\Gamma_{j}} \varphi_{h, 1} \boldsymbol{n} & \cdots & \int_{\Gamma_{j}} \varphi_{h, N} \boldsymbol{n}
\end{array}\right) \in \mathbb{R}^{2 \times N}, \quad j=1, \ldots, K .
$$

Then,

$$
\boldsymbol{M}_{\Gamma}:=\sum_{j=1}^{K} \frac{\rho_{0}}{k_{j}} \boldsymbol{B}_{j}^{\mathrm{t}} \boldsymbol{B}_{j} \in \mathbb{R}^{N \times N} \quad \text { and } \quad \boldsymbol{M}_{12}:=\left(\begin{array}{llll}
\frac{m_{1}}{k_{1}} \boldsymbol{B}_{1}^{\mathrm{t}} & \ldots & \frac{m_{K}}{k_{K}} \boldsymbol{B}_{K}^{\mathrm{t}}
\end{array}\right) \in \mathbb{R}^{N \times 2 K} .
$$

Notice that (6.1) corresponds to an eigenvalue problem posed on $\mathscr{W}_{h}^{p}$ and that the constraint $\int_{\Omega} u_{h}=0$ in the definition of $\mathscr{V}_{h}^{p}$ is not imposed. However, we show in what follows that there is no need of imposing it.

The symmetric matrix $\mathcal{K}$ is only positive semidefinite and

$$
\operatorname{ker} \mathcal{K}=\left\langle\boldsymbol{v}_{h}^{0}\right\rangle, \quad \text { where } \boldsymbol{v}_{h}^{0}=\left(\begin{array}{c}
\boldsymbol{u}_{h}^{0} \\
\mathbf{0}
\end{array}\right), \quad \text { with } \boldsymbol{u}_{h}^{0}:=\left(\begin{array}{c}
1 \\
\vdots \\
1
\end{array}\right) \in \mathbb{R}^{N} .
$$

In its turn, since $b$ is actually elliptic on the whole space $\mathscr{W}_{h}^{p}$, we have that the symmetric matrix $\mathcal{M}$ is positive definite. Therefore, (6.1) is a well posed generalized 
eigenvalue problem, which has $\boldsymbol{v}_{h}^{0}$ as an eigenvector corresponding to the eigenvalue $\omega_{h}^{2}=0$. All the other eigenvectors $\left(\boldsymbol{u}_{h}, \boldsymbol{s}_{h}\right)$ of problem (6.1) are $\mathcal{M}$-orthogonal to $\boldsymbol{v}_{h}^{0}$. This together with the facts that $\boldsymbol{M}_{\Gamma} \boldsymbol{u}_{h}^{0}=\mathbf{0}$ and $\boldsymbol{M}_{12}^{\mathrm{t}} \boldsymbol{u}_{h}^{0}=\mathbf{0}$ (which can be readily verified) lead to $\int_{\Omega} u_{h}=\boldsymbol{u}_{h}^{\mathrm{t}} \boldsymbol{M}_{\Delta} \boldsymbol{u}_{h}^{0}=0$. Therefore, all the eigenvectors of problem (6.1), except for $\boldsymbol{v}_{h}^{0}$, correspond to the eigenfunctions $\left(u_{h}, \boldsymbol{s}_{h}\right) \in \mathscr{V}_{h}^{\boldsymbol{p}} \times \mathbb{R}^{2 K}$ of problem (3.3).

In the incompressible case, the term $\frac{1}{c^{2}} \boldsymbol{M}_{\Delta}$ does not appear in (6.1). Thus, the matrix $\mathcal{M}$ is no longer positive definite and $\boldsymbol{v}_{h}^{0} \in \operatorname{ker} \mathcal{M}$, too (because of $\boldsymbol{M}_{\Gamma} \boldsymbol{u}_{h}^{0}=\mathbf{0}$ and $\boldsymbol{M}_{12}^{\mathrm{t}} \boldsymbol{u}_{h}^{0}=\mathbf{0}$ ). So, now (6.1) is not a well posed generalized eigenvalue problem. To obtain a well posed problem, a zero mean constraint was also assumed in this case for the analysis (cf. section 4). Alternatively, problem (3.3) can be posed in $\left(\mathscr{W}_{h}^{\boldsymbol{p}} / \mathbb{R}\right) \times \mathbb{R}^{2 K}$ instead of $\mathscr{V}_{h}^{\boldsymbol{p}} \times \mathbb{R}^{2 K}$. In such a case, since the vector of nodal components of functions in $\mathscr{W}_{h}^{p} / \mathbb{R}$ lies in $\mathbb{R}^{N} /\left\langle\boldsymbol{u}_{h}^{0}\right\rangle$, it is only necessary to fix any linear constraint not satisfied by $\boldsymbol{v}_{h}^{0}$. This is easily accomplished in practice by fixing one arbitrary nodal value of $u_{h}$ to zero, which leads to a positive definite left-hand matrix and, then, to a well posed problem.

6.2. Adaptive refinement strategy. We propose an adaptive scheme based on the error indicators $\eta_{T}$, an average strategy to mark the elements to be refined, and a variant of the approach from [9] to decide whether to proceed with an $h$ - or a $p$-refinement. A similar scheme has been introduced in [1] for another formulation of a particular case of problem (2.1) (incompressible fluid and the same parameters and geometry for all tubes). We report in Table 6.1 the resulting algorithm and refer to [1] and [9] for more details and the justification of each step of the marking and refinement procedure.

TABLE 6.1

Adaptive scheme algorithm

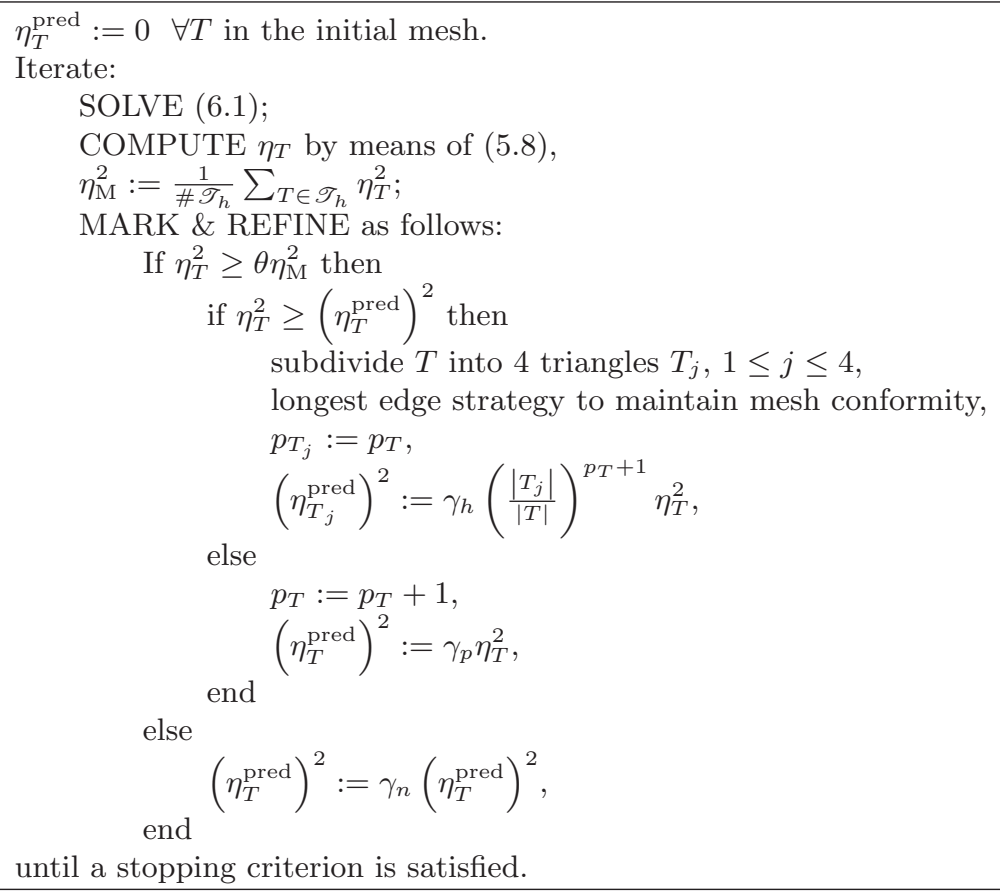


The algorithm in Table 6.1 relies on four positive parameters, $\theta, \gamma_{h}, \gamma_{p}$, and $\gamma_{n}$ that can be fixed by the user. We refer once more to [1,9] for further details.

7. A numerical test. The adaptive scheme analyzed in the previous sections has been designed with the goal of yielding optimal meshes for the computation of vibration modes corresponding to simple eigenvalues. However, in practice, it is very usual to have to deal with problems which have multiple eigenvalues because of the symmetry of the domain. This is the reason why we have chosen one such problem to show how the methodology described above can be used to solve it efficiently, too.

7.1. Exploiting the symmetry to deal with multiple eigenvalues. To illustrate the behavior of the adaptive scheme, we have applied it to compute the lowest-frequency vibration modes of the coupled system sketched in Figure 7.1. It consists of a square tube with side length $0.005 \sqrt{2} \mathrm{~m}$ centered in a quadrilateral fluidfilled cavity of side length $0.02 \mathrm{~m}$, as shown in Figure 7.1 (left).
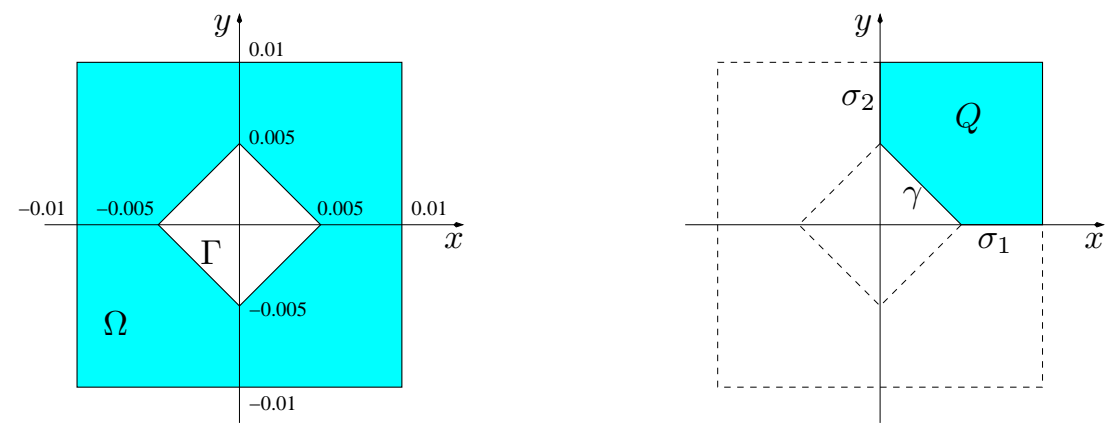

FIG. 7.1. Symmetric domain (left) and right-top quarter of the domain (right).

For the fluid, we have used physical parameters of water: $\rho_{0}=1000 \mathrm{~kg} / \mathrm{m}^{3}$ and $c=1493 \mathrm{~m} / \mathrm{s}$. For the tube we have used the parameters $m=0.22 \mathrm{~kg} / \mathrm{m}$ and $k=$ $27800 \mathrm{~N} / \mathrm{m}^{2}$, which have been taken from [12].

The two lowest-frequency vibration modes correspond to a unique eigenvalue of multiplicity two. A particular basis for the corresponding eigenspace can be chosen so that the tube moves in the vertical direction ("North-South") for one of the basis eigenmodes and in the horizontal direction ("East-West") for the other one. Accordingly, the velocity potential $u$ satisfies different symmetry conditions for each of these two vibration modes. For that in which the tube moves North-South, $u$ is symmetric with respect to the $y$-axis and antisymmetric with respect to the $x$-axis. The converse holds for the vibration mode in which the tube moves East-West.

Since the treatment of each of these two vibration modes is essentially identical, we have chosen for our test one of them: the mode involving North-South motion of the tube. In such a case, the tube velocity takes the form

$$
s:=\left(\begin{array}{l}
0 \\
s
\end{array}\right), \quad s \in \mathbb{R} .
$$

The symmetry of the problem leads us to consider a reduced problem posed on a quarter of the domain. We have chosen the right-top quarter $Q$ shown in Figure 7.1 (right); in what follows we use the notation introduced in this figure. According to the assumed motion of the tube, the fluid velocity potential has to satisfy the additional 
boundary conditions

$$
u=0 \quad \text { on } \sigma_{1} \quad \text { and } \quad \frac{\partial u}{\partial n}=0 \quad \text { on } \sigma_{2} .
$$

Let us remark that, in principle, the assumed symmetry only implies that the fluid velocity $\nabla u$ has to be orthogonal to $\sigma_{1}$, which in its turn implies that $\sigma_{1}$ has to be part of a level curve of $u$. However, since $\int_{\Omega} u=0$, necessarily $u$ has to vanish on $\sigma_{1}$.

Reducing problem (2.1) to the domain $Q$ is not quite straightforward because of the non-local character of the term $\int_{\Gamma} u \boldsymbol{n}$. To derive the correct problem on $Q$, we write the variational formulation (2.4) in terms of quantities restricted to this domain. In particular, for $u$ and $v$ being symmetric with respect to the $y$-axis and antisymmetric with respect to the $x$-axis, we have the following relations:

$$
\begin{array}{ll}
\int_{\Omega} \nabla u \cdot \nabla v=4 \int_{Q} \nabla u \cdot \nabla v, & \int_{\Omega} u v=4 \int_{Q} u v, \\
\int_{\Gamma} u \boldsymbol{n}=\left(\begin{array}{c}
0 \\
4 \int_{\gamma} u n_{2}
\end{array}\right), & \int_{\Gamma} v \boldsymbol{n}=\left(\begin{array}{c}
0 \\
4 \int_{\gamma} v n_{2}
\end{array}\right) .
\end{array}
$$

Therefore, using these relations, (7.1), and the analogue for the test function, $\boldsymbol{t}=\left(\begin{array}{l}0 \\ t\end{array}\right)$, we are lead to the following problem:

Find $\omega>0$ and non-vanishing $(u, s) \in H_{\sigma_{1}}^{1}(Q) \times \mathbb{R}$ such that

$$
4 \int_{Q} \nabla u \cdot \nabla v+\frac{m}{\rho_{0}} s t=\omega^{2}\left[\frac{4}{c^{2}} \int_{Q} u v+\frac{\rho_{0}}{k}\left(4 \int_{\gamma} u n_{2}+\frac{m}{\rho_{0}} s\right)\left(4 \int_{\gamma} v n_{2}+\frac{m}{\rho_{0}} t\right)\right]
$$

for all $(v, t) \in H_{\sigma_{1}}^{1}(Q) \times \mathbb{R}$, where $H_{\sigma_{1}}^{1}(Q):=\left\{v \in H^{1}(Q):\left.v\right|_{\sigma_{1}}=0\right\}$.

The theoretical framework developed in the previous sections extends readily to this problem.

7.2. Numerical results. The uniform coarse mesh shown in Figure 7.2 (left) has been used to initiate the adaptive process with quadratic finite elements in all triangles. The color palette used in this and all forthcoming meshes indicates the polynomial degree of each element.
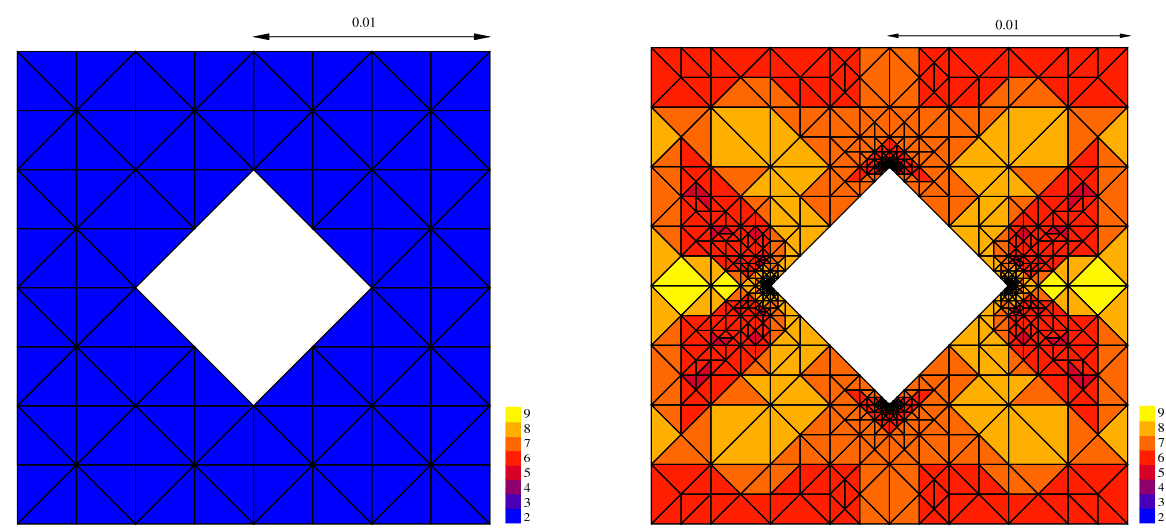

FIG. 7.2. Domain and initial mesh (left) and refined mesh at step 25 for the lowest-frequency vibration mode with North-South motion of the tube (right) 
Although we actually solve the problem in a quarter of the domain, for an easier visualization we show the corresponding results in the whole domain. In particular, Figure 7.2 (right) shows the resulting refined mesh at step 25.

In this test, the fluid domain has reentrant angles at the vertices of the tube. Because of this, the computed eigenfunction is singular at these four points. However, the singularity strength at the Northern-Southern vertices differ from that at the Eastern-Western ones. The behavior of the adaptive scheme in the neighborhood of the two different singularities can be appreciated from Figures 7.3 and 7.4.
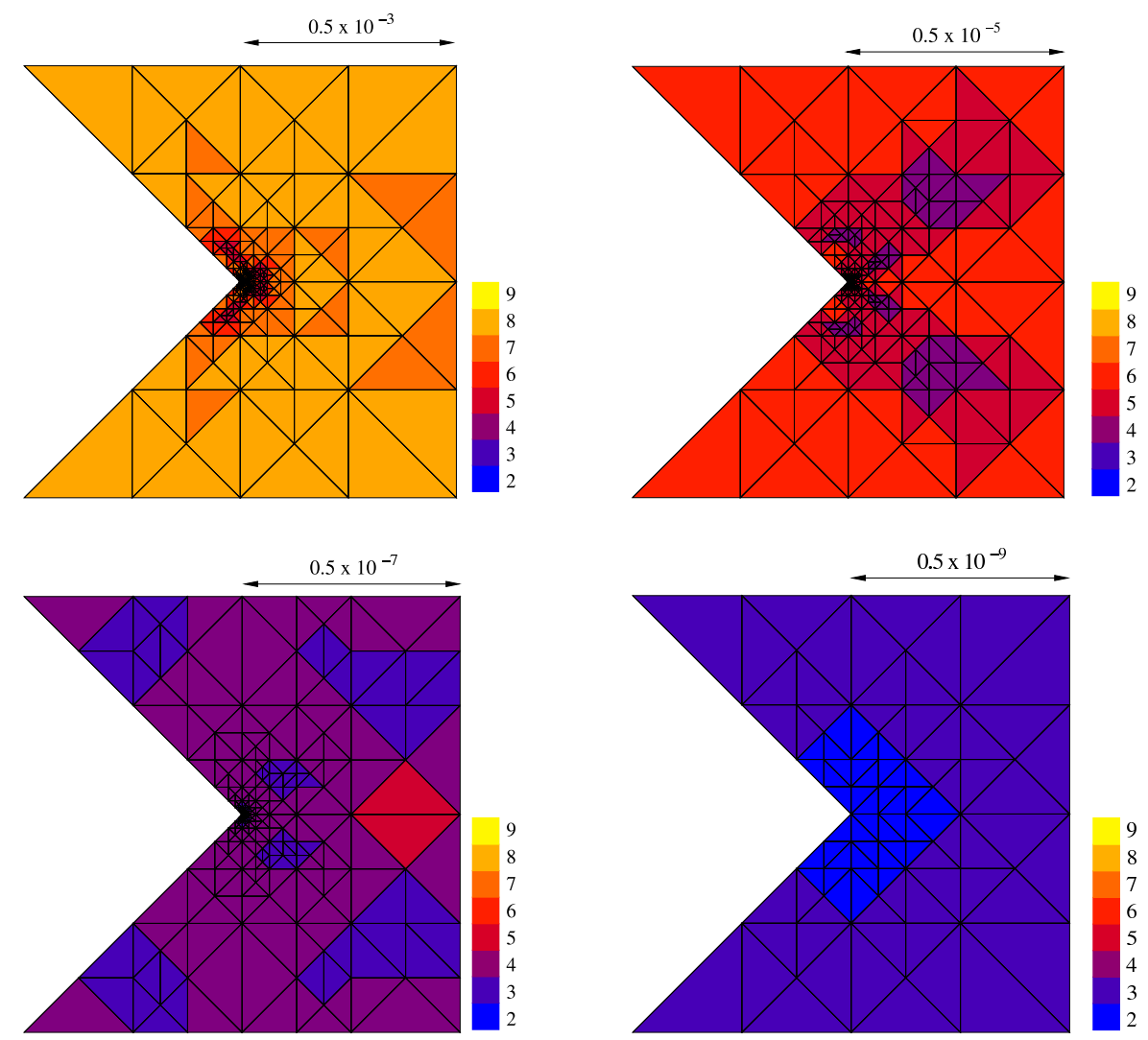

FIG. 7.3. Lowest-frequency vibration mode with North-South motion of the tube. Successive zooms at the Eastern vertex of the refined mesh at step 25.

These figures show sequences of zooms of the mesh at step 25 around two different singular points: the Eastern (Figure 7.3) and the Northern (Figure 7.4) vertices of the tube. The last zoom in Figure 7.3 enlarges the mesh $10^{7}$ times, while the last zoom in Figure 7.4 enlarges it only $10^{4}$ times. Therefore, we observe from these figures that the smallest elements near the Eastern vertex are approximately 1000 times smaller than those around the Northern vertex. This indicates that, in this case, the strongest singularities appear at the Eastern-Western vertices of the square. Moreover, in both cases, we observe the typical $h p$ adaptive behavior: the closer to the singularity, the more dominant the $h$-refinement is. In particular there is no $p$-refinement at all in the elements nearest the strongest singularity.

It is known (see, for instance, [3]) that a proper combination of $h$ - and $p$-refinement allows obtaining an exponential rate of convergence in terms of a fractional power of 

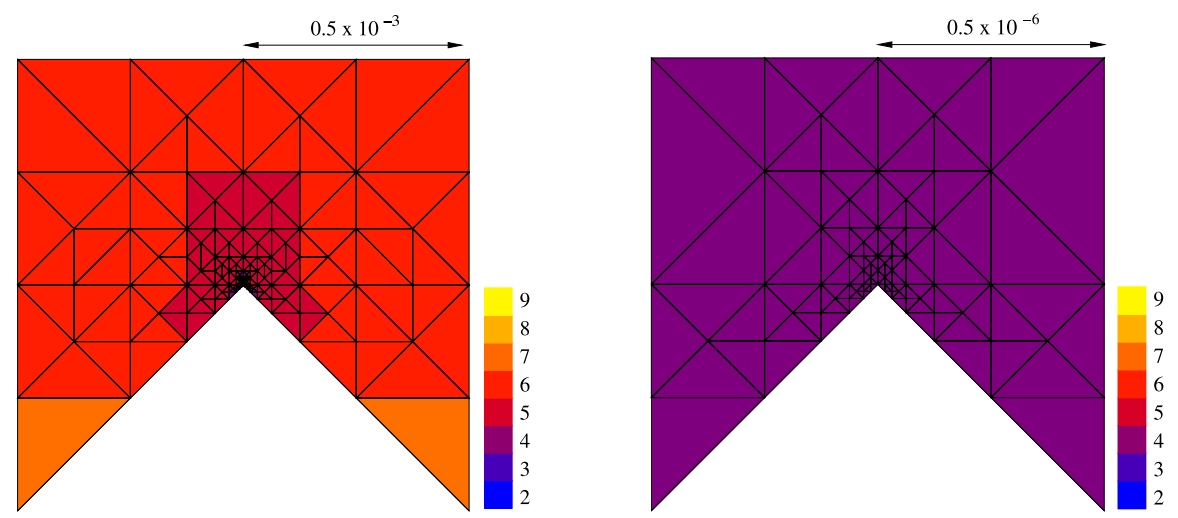

FIG. 7.4. Lowest-frequency vibration mode with North-South motion of the tube. Successive zooms at the Northern vertex of the refined mesh at step 25.

the number $N$ of degrees of freedom in the finite element mesh. We use the same vibration mode as above to investigate this rate of convergence. Figure 7.5 (left) shows a plot of $\log \eta_{\Omega}$ versus $\sqrt[3]{N}$, which allows us to observe that the estimated error $\eta_{\Omega}$ behaves asymptotically as follows:

$$
\eta_{\Omega} \approx \kappa_{1} e^{-\alpha \sqrt[3]{N}}
$$
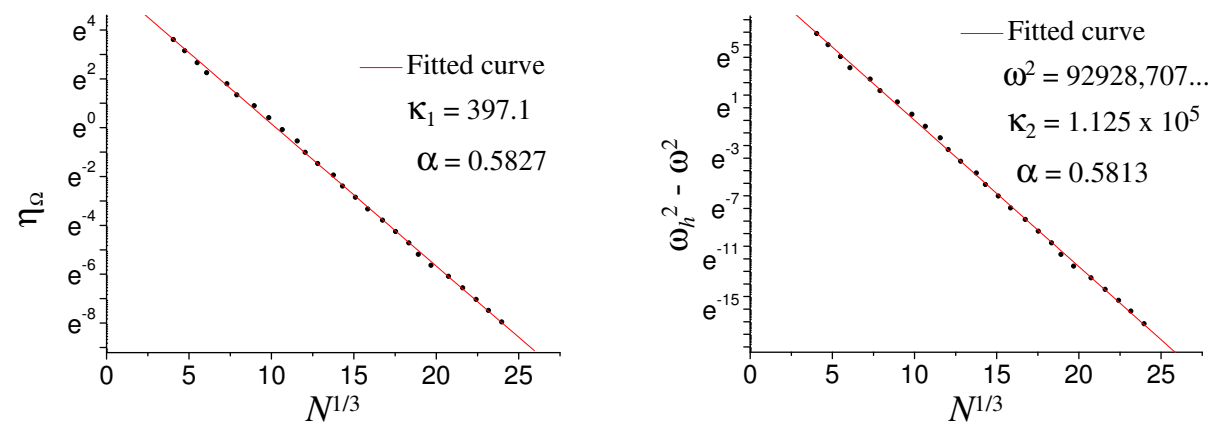

FIG. 7.5. Lowest-frequency vibration mode with North-South motion of the tube. Error curves: $\eta_{\Omega}$ (logarithm scale) versus $\sqrt[3]{N}$ (left) and $\omega_{h}^{2}-\omega^{2}$ (logarithm scale) versus $\sqrt[3]{N}$ (right).

No analytical solution is available in this case to check whether the actual error also attains an exponential rate of convergence. To provide some numerical evidence of such a behavior, we have estimated the error of the computed eigenvalues by using as 'exact' a more accurate approximation obtained by an extrapolation procedure. To do this, we have used the fact that the computed eigenvalues are expected to converge with double order and we have determined the parameters $\omega^{2}, \kappa_{2}$, and $\alpha$ in the model

$$
\omega_{h}^{2} \approx \omega^{2}+\kappa_{2} e^{-2 \alpha \sqrt[3]{N}}
$$

by means of a weighted least-squares fitting. The weights have been chosen so that the more accurate the computed values $\omega_{h}$ (i.e., the larger the number of degrees 
of freedom of the used mesh), the more significant the role in the fitting. Since we found that the residuals of these meshes are approximately proportional to $e^{-2 \alpha \sqrt[3]{N}}$, we have chosen the weights equal to $e^{4 \alpha \sqrt[3]{N}}$. Thus, we have obtained a fitted value $\omega^{2}=92928.70795816$ which we have used to plot $\log \left(\omega_{h}^{2}-\omega^{2}\right)$ versus $\sqrt[3]{N}$. This plot is shown in Figure 7.5 (right), where a linear dependence can be clearly seen for sufficiently large values of $N$.

The computed values of $\alpha$ in both fittings are quite close: $\alpha=0.5827$ in the former and $\alpha=0.5813$ in the latter. This excellent agreement provides a sound numerical evidence of the fact that the error estimate behaves asymptotically as the actual error. Such convergence behavior is coherent with the theoretically expected exponential decay of the error with respect to the number of degree of freedom. In fact, the estimated convergence rated $e^{-\alpha \sqrt[3]{N}}$ is typical for the $h p$ version of the finite element method for source elliptic problems with piecewise analytical data in the presence of corner singularities (cf. [3]). Let us remark that this convergence rate does not agree with what was reported in a previous paper [1], where an improved rate $e^{\alpha \sqrt{N}}$ seemed to be observed for another formulation applied to a similar test.

We report in Table 7.1 the ten lowest vibration frequencies of this test problem. Each of them has been computed by means of a particular execution of the adaptive scheme and a subsequent least squares fitting as described above. In all cases, we have used formulations reduced to a quarter of the domain analogous to (7.2), which result from taking advantage of the different symmetry conditions of each corresponding eigenfunction.

TABLE 7.1

Lowest vibration frequencies

\begin{tabular}{cc}
\hline Mode & $f(\mathrm{~Hz})$ \\
\hline 1 & 48.51712761 \\
2 & 48.51712761 \\
3 & 32810.71079 \\
4 & 32810.71079 \\
5 & 49255.90150 \\
6 & 71587.34925 \\
7 & 82786.44265 \\
8 & 82786.44265 \\
9 & 85852.07346 \\
10 & 105947.55960 \\
\hline
\end{tabular}

We observe in Table 7.1 a significant gap between the first (double) vibration frequency and the rest of the spectrum. The two lowest-frequency vibration modes (which are the most relevant in practice) correspond to vibrations of the tube modified by the presence of the fluid, which behaves as almost incompressible. This eigenfrequency depend strongly on the coefficients $k$ and $m$ of the tube and only very slightly on those of the fluid, $\rho_{0}$ and $c$. In this case, the fluid motion is induced as response to the movement of the tube. All the other eigenfrequencies of this test correspond to acoustic vibration modes of the fluid; they are almost proportional to the sound speed $c$ and depend only slightly of the physical parameters of the tube. In this case, it is the tube which moves as a consequence of the difference of pressure exerted by the fluid on its walls. Figures 7.6 and 7.7 show the velocity fields of the fluid and the tube for the four lowest-frequency vibration modes. Each of these figures shows two linearly independent eigenmodes corresponding to a double eigenvalue (see Table 7.1). 
Notice the symmetry of the velocity fields at left and right of each figure.
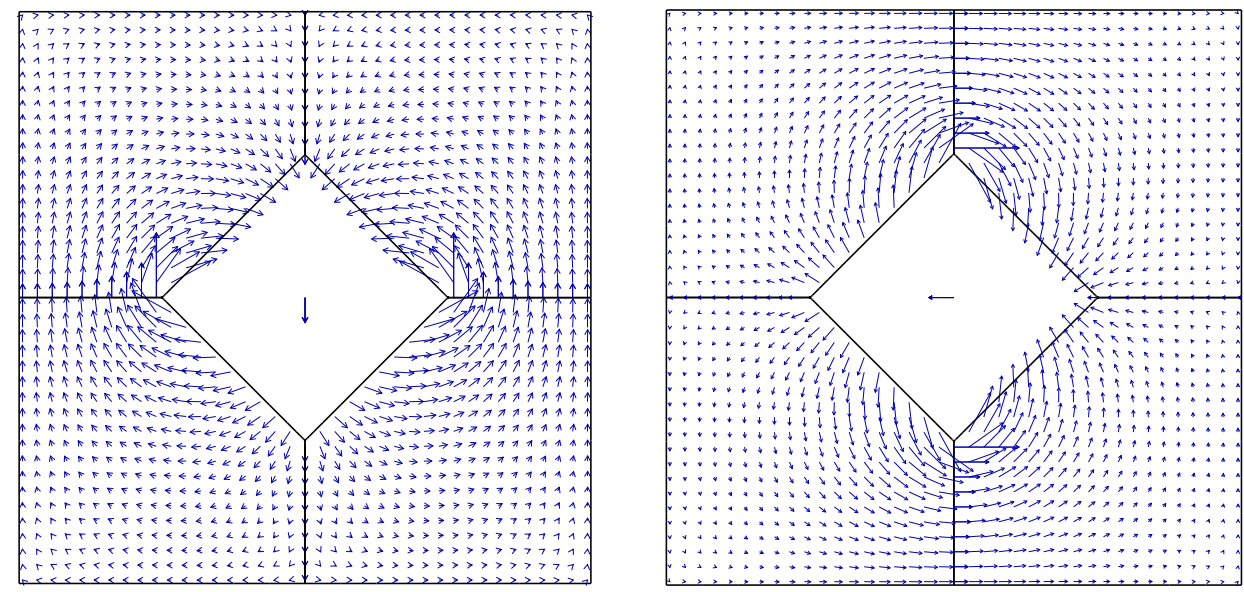

FIG. 7.6. Velocity fields of the lowest-frequency vibration modes with North-South (left) and East-West (right) motion of the tube.
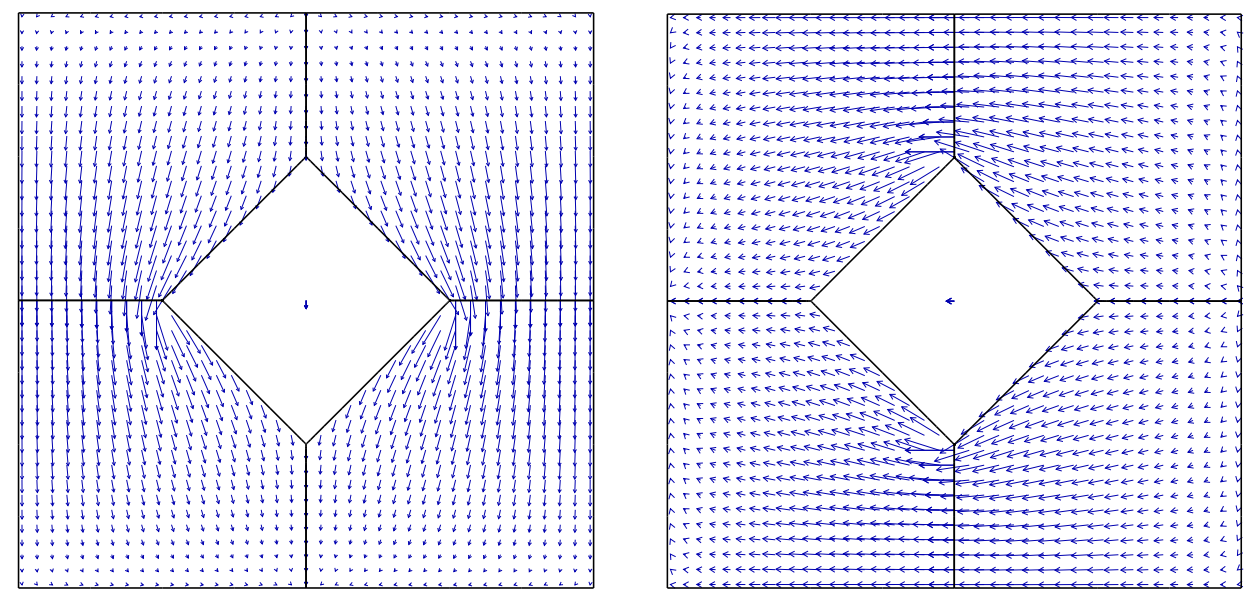

FIG. 7.7. Velocity fields of the second lowest-frequency vibration modes.

On the other hand, as shown in section 4, the numerical method is also applicable to perfectly incompressible fluids. To test the adaptive scheme in this case, we have used the same problem but with "incompressible water"; namely, a fluid with the same density as water, $\rho_{0}=1000 \mathrm{~kg} / \mathrm{m}^{3}$, but sound speed $c=\infty$. In this case, there is only one (double) eigenfrequency, since all the acoustic modes blow up as $c$ goes to $\infty$. The computed vibration frequency is $f_{\infty}=48,51713814 \mathrm{~Hz}$. This value agrees with that obtained in [1] with an alternative formulation valid only for incompressible fluids applied to the same test problem. Notice how close is $f_{\infty}$ to the first vibration frequencies reported in Table 7.1. This justifies modeling in this case the fluid as incompressible. On the contrary, the presence of the fluid in the model plays a significant role, as can be seen from the fact that the vibration frequency of the tube in absence of fluid is significantly larger: $f_{\emptyset}:=\frac{1}{2 \pi} \sqrt{\frac{k}{m}}=56.57586324 \mathrm{~Hz}$. 


\section{REFERENCES}

[1] M.G. Armentano, C. Padra, R. Rodríguez, and M. Scheble, An hp finite element adaptive scheme to solve the Laplace model for fluid-solid vibrations, Comput. Methods Appl. Mech. Engrg., 200 (2011) 178-188.

[2] I. Babuška and J. Osborn, Eigenvalue Problems, in Handbook of Numerical Analysis, Vol. II, P.G. Ciarlet and J.L. Lions, eds., North-Holland, Amsterdam, 1991, pp. 641-787.

[3] I. Babuška and M. Suri, The $p$ and $h-p$ versions of the finite element method, basic principles and properties, SIAM Review 36 (1994) 578-632.

[4] C. Conca, J. Planchard, and M. Vanninathan, Fluid and Periodic Structures, Masson, Paris, 1995.

[5] L. Demkowicz, Computing with hp-adaptive Finite Elements. Vol 1. One and Two Dimensional Elliptic and Maxwell Problems, Chapman \& Hall/CRC, Boca Raton, FL, 2007.

[6] J.P. Grégoire, J.C. Nédélec, and J. Planchard, A method of finding eigenvalues and eigenfunctions of self-adjoint operators, Comput. Methods. Appl, Mech. Engrg., 8 (1976) 201-214.

[7] P. Grisvard, Elliptic Problems in Nonsmooth Domain, Pitman, Boston, 1985.

[8] J.M. Melenk, hp-finite element methods for singular perturbations, Lecture Notes in Mathematics, 1796. Springer-Verlag, Berlin, 2002.

[9] J.M. Melenk and B.I. Wohlmuth, On residual-based a posteriori error estimation in hp-FEM, Adv. Comput. Math., 15 (2001) 311-331.

[10] H.J.-P. Morand and R. Ohayon, Fluid-Structure Interaction, John Wiley \& Sons, Chichester, 1995.

[11] J. Planchard, Eigenfrequencies of a tube bundle placed in a confined fluid, Comput. Methods Appl. Mech. Engrg., 30 (1983) 75-93.

[12] J. Planchard and M. Ibnou-Zahir, Natural frequencies of tube bundle in an uncompressible fluid, Comput. Methods Appl. Mech. Engrg., 41 (1983) 47-68.

[13] P.A. Raviart and J.M. Thomas, Introduction à l'Analyse Numérique des Equations aux Dérivées Partielles, Masson, Paris, 1983. 